\title{
MATHEMATICAL THEORY OF MEDIAL AXIS TRANSFORM
}

\author{
Hyeong In Choi, Sung Woo Choi and Hwan Pyo Moon
}

The medial axis of a plane domain is defined to be the set of the centers of the maximal inscribed disks. It is essentially the cut loci of the inward unit normal bundle of the boundary. We prove that if a plane domain has finite number of boundary curves each of which consists of finite number of real analytic pieces, then the medial axis is a connected geometric graph in $\mathbb{R}^{2}$ with finitely many vertices and edges. And each edge is a real analytic curve which can be extended in the $C^{1}$ manner at the end vertices. We clarify the relation between the vertex degree and the local geometry of the domain. We also analyze various continuity and regularity results in detail, and show that the medial axis is a strong deformation retract of the domain which means in the practical sense that it retains all the topological informations of the domain. We also obtain parallel results for the medial axis transform.

\section{Introduction.}

One of the difficult problems in global differential geometry is the precise description and the exact determination of the cut loci of a set. It is because a cut locus arises as a critical point of a certain distance function, and studying it is in general a nontrivial problem.

In this paper, we study the following version: Let $\Omega$ be a connected bounded domain in $\mathbb{R}^{2}$. Let $\operatorname{CORE}(\Omega)$ be the set of the maximal inscribed disks in $\Omega$. We define the medial axis, denoted by $\mathbf{M A}(\Omega)$, to be the set of the centers of the disks in $\operatorname{CORE}(\Omega)$. Thus if $\Omega$ has smooth boundary, $\mathbf{M A}(\Omega)$ is essentially the set of the cut loci of the inward pointing unit normal bundle of $\partial \Omega$. But since we allow $\Omega$ to have corners, our case includes a slightly more general notion. (Consult Definition 4.4 and its subsequent role.) We can also define the medial axis transform, denoted by $\mathbf{M A T}(\Omega)$, to be the set of the pairs consisting of the center and the radius of the disks in $\operatorname{CORE}(\Omega)$.

The medial axis is a continuous version of the so-called Voronoi diagram which was introduced by Voronoi [14]. Voronoi diagram was originally defined for a finite set of points in $\mathbb{R}^{2}$, and in fact this and the related notions can be traced as far back as to Dirichlet [3]. 
Although the medial axis is a natural geometric object, its careful mathematical study has been lacking in the pure mathematics communities. However, the medial axis transform has been widely used in the engineering and the computer science community. The modern incarnation of the medial axis transform was introduced by Blum [1] to extract and represent the salient features of a planar shape (domain). Since then, there has been a prolific amount of literatures in many application areas such as vision, pattern recognition, NC tool path planning, and FEM mesh generation, and so on. However, the engineering and the computer science communities are mainly interested in the algorithmic aspects of actually finding the medial axis transform under specific conditions on the domain, and they seem to be less interested in pinning down the intricate mathematical details for general class of domains. Most of the known results deal with the domain with the boundary consisting of line segments and pieces of circular arcs. These can be found in $[5,8,9,10,13,16]$, to name a few.

However, even in the application areas, the boundary curves need to be of much more general form. The kind of curve representation accepted as the most general in application is called the NURBS, i.e., Non-Uniform Rational B-Splines. Mathematically, they arise as splines in the projective geometry, and they are all rational functions of the curve parameter, But, in this generality, not so much is known for the medial axis transform. Our paper with N.-S. Wee [2] gives a good approximate algorithm which works well in practical situations with very general boundary curve assumptions, i.e., boundary is assumed to be composed of finite number of real analytic curves. In proving that our algorithm works and terminates in finite steps, etc., we had to have the rigorous mathematical foundation. As we pondered over these issues, we discovered many interesting pieces of mathematics. This paper is an outgrowth of that.

One important observation that is relevant to our subsequent analysis is the regularity assumption of the domain. Contrary to common belief, it is not enough to assume that the boundary curve is $C^{\infty}$. In fact, we show that there are plenty of domains with $C^{\infty}$ boundary that have pathological behavior. For example, there are domains with $C^{\infty}$ boundary that have infinitely many inscribed osculating circles, or infinitely many bifurcation circles. Or, they may have a medial axis point from which infinitely many branches of the medial axis emanate. These may make the medial axis an infinite graph. In fact, it is possible to create all kinds of pathological examples with the $C^{\infty}$ assumption on the boundary.

Thus we have to restrict the class of domains in order to do meaningful analysis. The domains we consider are the ones with finitely many boundary curves each of which consists of finite number of real analytic pieces. The 
real analytic pieces in the boundary may meet with each other in the $C^{1}$ manner, or may meet at a corner. This kind of restrictions pose no real loss in most practically important situations, since almost all domains that have practical importance have this property. For instance, the boundary curves may be represented as NURBS curves which fall into this category. We cannot help believing that our assumption is the most natural and optimal in practice.

The basic strategy of our analysis is a judicious combination of local differential geometry and some of the tools we develop. The most important tool is the Domain Decomposition Lemma which enables us to break up the domain into simpler pieces, thus localizing the analysis. We also do careful analysis relating the curvature. We then prove various finiteness results: the finiteness of the inscribed osculating circles and the bifurcation circles; and the finiteness of the number of the branches of the medial axis of the domain emanating from any medial axis point. These analyses enable us to obtain various continuity and regularity results. Combining all these together, we prove that the medial axis is a connected geometric graph in $\mathbb{R}^{2}$ with finitely many vertices and edges, and each edge is a real analytic curve which can be extended in the $C^{1}$ manner at the end vertices. We show that the medial axis is a strong deformation retract of the domain which means in the practical sense that it retains all the topological informations of the domain. We also obtain parallel results for the medial axis transform as a geometric graph in $\mathbb{R}^{3}$.

A word on related works is in order. After this paper was submitted, we received three papers which are relevant to our work: In [15], F.-E. Wolter also proved that the medial axis is a strong deformation retract of the domain, although his method of proof is different from ours. Sherbrooke, Patrikalakis and Wolter [11] also obtained the same result using different method. In [6], Hoffmann and Chiang discuss various aspects of the domain decomposition lemma. In fact, they essentially obtained the same result when the domain is simply connected but with no dull corners. They also indicate how to decompose the domain when it has homology. But their idea, although very close in spirit, is not exactly in the form of our domain decomposition lemma. The paper [15] of Wolter contains many interesting results related to this work. For example, he gives interesting discussions on the cut locus, equidistance set, Voronoi diagram, and the medial axis from general view point. He also obtained other related topological results. An interested reader should consult his paper [15].

We gratefully acknowledge the support of Hyundai Media Systems Co., Ltd. Special thanks are due to Dr. Jin-Kee Lee, and Directors Joon W. Kim and Tae-Won Kang. We would like to thank Dr. Sungjin Lee and Kyung 
Hwan Park for their interest in our work, and Sun Gi Hong, Director of R\&D of Microsoft Korea, for the support over the years. Finally, we would also like to thank Heungju Ahn for pointing out the paper [7].

\section{Standing Assumption on Domain.}

Before we proceed further, we need to restrict the class of the domains for our analysis. In doing so, the important requirement is that it must be inclusive enough to cover almost all practically important cases, while at the same time restrictive enough to be amenable to thorough analysis.

First, let us look at some examples with pathological behavior even with $C^{\infty}$ boundary.

Example 2.1. Let $\Omega$ be the domain whose boundary consists of two $C^{\infty}$ curves $\alpha$ and $\beta$, where $\alpha$ is an arc portion of the unit circle $\{\zeta \in \mathbb{C}:|\zeta|=1\}$ and $\beta$ is a curve represented by

$$
\beta(\theta)=\left(1+e^{-1 / \theta^{2}} \sin ^{2} \frac{1}{\theta}\right) e^{i \theta},
$$

for sufficiently small $|\theta|$, and $\alpha$ and $\beta$ are joined in such a way as in Figure 1 to form a closed $C^{\infty}$ curve.

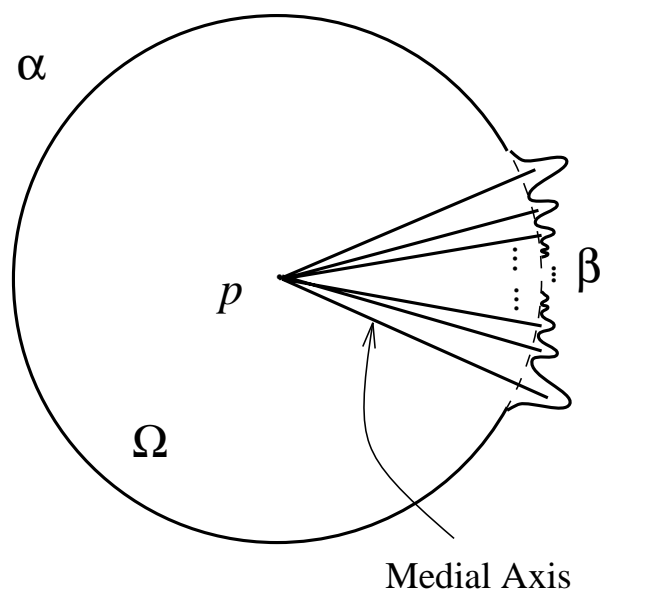

Figure 1. Medial Axis Point with Infinitely Many Prongs.

Then it is easy to see that the point $p$ is in the medial axis of $\Omega$ and there are infinitely many prongs in the medial axis of $\Omega$ emanating from $p$.

Example 2.2. Let $\Omega$ be the domain whose boundary consists of two $C^{\infty}$ curves $\gamma$ and $\delta$, where $\gamma$ is a portion of the boundary of a stadium and $\delta$ is 
a curve represented by

$$
\delta(t)=\left(t, e^{-1 / t^{2}} \sin ^{2} \frac{1}{t}\right)
$$

for sufficiently small $|t|$, and $\gamma$ and $\delta$ are joined in such a way as in Figure 2 to form a closed $C^{\infty}$ curve.

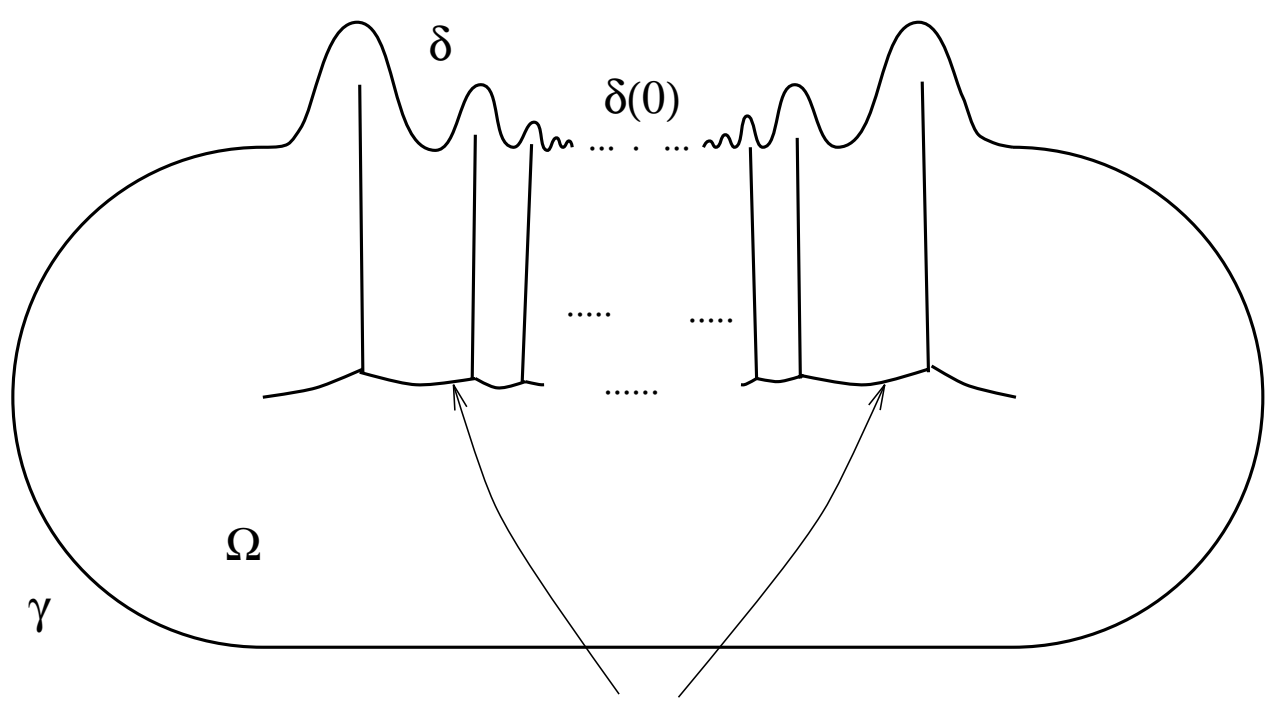

Medial Axis

Figure 2. Medial Axis having Infinitely Many Bifurcation Points.

Then it is easy to see that the medial axis of $\Omega$ has infinitely many bifurcation points.

The situations in the above examples are bad, because these should not happen in real applications, and such kind of pathological phenomena make algorithm unnecessarily complicated, and often times it is very difficult to design practically workable algorithms to capture these phenomena.

After some analysis, we concluded that the most natural domains must be the ones that have finite number of boundary curves each of which consists of finite number of real analytic pieces. Once our condition is violated, one can easily cook up some counterexamples to many of the important results in this paper. However, these kinds of pathologies are not meaningful in real world applications.

Definition 2.1. A curve $\gamma:(a, b) \rightarrow \mathbb{R}^{n}(n=1,2, \ldots)$, is a $C^{k}$ curve, if there is a reparametrization $\bar{\gamma}(t)=\left(x_{1}(t), \ldots, x_{n}(t)\right)$ of $\gamma$ by the arc length, 
such that $x_{1}(t), \ldots, x_{n}(t)$ are $C^{k}$ functions of $t$, where $k=1,2, \ldots, \infty$, or $k=\omega$ when it is real analytic. A curve $\gamma:[a, b] \rightarrow \mathbb{R}^{n}(n=1,2, \ldots)$ is a $C^{k}$ curve, if there exists a $C^{k}$ curve $\tilde{\gamma}:(a-\epsilon, b+\epsilon) \rightarrow \mathbb{R}^{n}$ for some $\epsilon>0$ such that $\tilde{\gamma}(t)=\gamma(t)$ for all $t \in[a, b]$, where $k=1,2, \ldots, \infty, \omega$. A $C^{\omega}$ curve will also be called a real analytic curve.

Remark 2.1. A curve $\gamma:(a, b) \rightarrow \mathbb{R}^{n}(n=1,2, \ldots)$, is a $C^{k}$ curve in the sense of the above definition, if and only if, for any point $p$ on $\gamma$, there exists a small number $\epsilon$ such that the curve $\gamma$ can be represented as the graph of a $C^{k}$ function with respect to the tangent line at $p$ inside the $\epsilon$-disk $B_{\epsilon}(p)$ in $\mathbb{R}^{n}$, where $k=1,2, \ldots, \infty, \omega$.

We now list the assumptions which a domain $\Omega$ in our class should satisfy.

Assumption 1. $\Omega$ is the closure of a connected bounded open subset in $\mathbb{R}^{2}$ bounded by finite number of mutually disjoint simple closed curves. (Here a simple closed curve means an embedding of the unit circle in $\mathbb{R}^{2}$.)

The closedness and the connectedness of domain simplifies much of the technical arguments; and the boundedness is not only appropriate for practical settings, but also essential sometimes in our analysis of the medial axis transform, since, it, together with the closedness, implies the compactness. The simple closed curve in $\partial \Omega$ which bounds the unbounded connected component of $\mathbb{R}^{2} \backslash \Omega$, is called the outer boundary curve, and the rest of the simple closed curves in $\partial \Omega$ are called the inner boundary curves. The number of the inner boundary curves in $\partial \Omega$ is called the genus of $\Omega$.

Assumption 2. Each simple closed curve in $\partial \Omega$ consists of finite number of pieces of real analytic curves.

Let us be more clear about this assumption: Assumption 2 means that each simple closed curve in $\partial \Omega$ is represented as a closed curve $\gamma:[a, b] \rightarrow \mathbb{R}^{2}$ such that there exist finite number of points $a=t_{0}<t_{1}<\cdots<t_{N}=b \mathrm{such}$ that $\gamma_{\left[t_{i-1}, t_{i}\right]}$ is a real analytic curve for $i=1, \ldots, N$. We call $\gamma_{\left[t_{i-1}, t_{i}\right]}$ a real analytic piece (of $\gamma$ ). It should be noted that $\gamma$ is $C^{1}$ at a point where two real analytic pieces join as long as the unit tangent vector fields along each piece coincide at that point. However, it is not realistic to expect it to be $C^{2}$ in general because the curvature of the two pieces may not coincide at the joint point. But, assuming each piece should be a polynomial curve is too restrictive, because, for example, conic sections, such as ellipses, parabolas, or hyperbolas, which are frequently used as NURBS curves are in general not polynomial curves. (Note that their $\mathrm{x}$ and $\mathrm{y}$ coordinates may have a polynomial relation, but the $\mathrm{x}$ and $\mathrm{y}$ coordinates of these conic sections cannot be polynomial functions of some parameter simultaneously.) Thus 
the right kinds of general class of curves are real analytic pieces joined in the $C^{1}$ manner except at corner points.

Standing Assumption. From now on, by the term domain, we mean a non-circular domain satisfying the above two assumptions unless otherwise stated.

The reason we usually exclude the circular domain, i.e., the disk, is that the disk poses an exception to many of our technical results. But since it is a trivial domain with the medial axis consisting of one point, everything is known. Thus there is no real loss of generality in excluding the disk.

\section{Curvature and Inscribed Osculating Circle.}

In this section, we present some preliminaries about curvature and osculating circle. First let us fix the notations about the curvature.

Definition 3.1. Let $\gamma:[a, b] \rightarrow \mathbb{R}^{2}$ be a $C^{2}$ curve parameterized by the arc-length. The (geodesic) curvature of $\gamma$ is defined to be $\left\langle\gamma^{\prime \prime}, N\right\rangle$, where $N$ is the unit vector obtained by rotating $\gamma^{\prime}$ counterclockwise by 90 degrees.

Here, $\langle$,$\rangle denotes the standard inner product in \mathbb{R}^{2}$.

Remark 3.1. In fact, we don't need the arc length parametrization to define the (geodesic) curvature, since it is an intrinsic quantity. So we can freely use the term (geodesic) curvature for any $C^{2}$ curve, even if it is not parametrized by the arc length.

Let $\gamma:[a, b] \rightarrow \mathbb{R}^{2}$ be a $C^{2}$ curve. We call a circle $C$ centered at $p$ an osculating circle of $\gamma$ at $\gamma(t)(t \in[a, b])$, if the following conditions are satisfied.

(1) $\gamma(t)$ is on $C$.

(2) The tangent line of $C$ at $\gamma(t)$ is tangent to $\gamma$.

(3) $\overrightarrow{\gamma(t) p}$ is in the direction of $N$ (resp., $-N$ ), if the curvature of $\gamma$ at $\gamma(t)$ is positive (resp., negative).

(4) The radius of $C$ is equal to $1 /|\kappa(t)|$.

Definition 3.2. Let $\gamma:(a, b) \rightarrow \mathbb{R}^{2}$ be a $C^{2}$ curve, and let $p$ be a point on the half line from $\gamma\left(t_{0}\right)\left(t_{0} \in(a, b)\right)$ with the direction of $N$, where $N$ is defined as above. Then we say that $p$ is within the focal locus of $\gamma$ near $\gamma\left(t_{0}\right)$, if

$$
\frac{1}{d\left(p, \gamma\left(t_{0}\right)\right)}>\kappa\left(t_{0}\right)
$$


Now we fix the sign of the curvatures of the points in the boundary of a domain. Let $p$ be a point in $\partial \Omega$, and let $\gamma:(-\epsilon, \epsilon) \rightarrow \partial \Omega$ be a curve such that $\gamma(0)=p$, where $\gamma$ is oriented in such a way that the interior of $\Omega$ is always on the left of $\gamma$. Then the we denote $\kappa^{+}(p)=\lim _{t \rightarrow 0+} \kappa(t)$ and $\kappa^{-}(p)=\lim _{t \rightarrow 0-} \kappa(t)$, where $\kappa$ is the geodesic curvature of $\gamma$. Note that this is well-defined because of our assumption on $\partial \Omega$, and does not depend on the choice of $\gamma$. If $\kappa^{+}(p)=\kappa^{-}(p)$, we denote $\kappa(p)=\kappa^{+}(p)\left(=\kappa^{-}(p)\right)$ and call $\kappa(p)$ the (geodesic) curvature at $p$.

We need the following lemma for the treatment of the osculating circles.

Lemma 3.1. Let $I$ be an interval of the type $[0, \delta]$ or $[-\delta, 0]$. Let $\gamma: I \rightarrow$ $\mathbb{R}^{2}$ be a $C^{2}$ curve. Let $\kappa$ be its geodesic curvature function. Assume $\kappa(0) \geq 0$. Suppose $\kappa(t)>\kappa(0)$ (resp., $\kappa(t)<\kappa(0))$ for all $t \in I \backslash\{0\}$. Then, for sufficiently small $\delta, \gamma(t)$, for $t \neq 0$, must lie strictly inside (resp., outside) the osculating circle of $\gamma$ at $\gamma(0)$ when $\kappa(0)>0$. And, when $\kappa(0)=0$, for sufficiently small $\delta, \gamma(t)$, for $t \neq 0$, must lie strictly in the half plane $P=\left\{p \in \mathbb{R}^{2} \mid\langle\overrightarrow{\gamma(0) p}, N(0)\rangle>(\right.$ resp.,$\left.<) 0\right\}$, where $N$ is the usual counterclockwise rotation of $\gamma^{\prime}$ by 90 degrees.

Proof. Let us first give the proof for the case when $\kappa(t)>\kappa(0)$. In the proof we regard $\mathbb{R}^{2}$ as the complex plane $\mathbb{C}$, and points and vectors are also regarded as complex numbers. Let us first give the proof for $I=[0, \delta]$. Suppose $\kappa(0)>0$. By rescaling, we may assume without loss of generality that $\kappa(0)=1$. We may also assume without loss of generality that $\gamma$ is parametrized by the arc-length $s$. Let $T(s)=\gamma^{\prime}(s)$. Then $N(s)=i T(s)$, and

$$
T^{\prime}(s)=\kappa(s) N(s)=i \kappa(s) T(s) .
$$

Dividing both sides by $T(s)$, and integrating, we have

$$
T(s)=T(0) e^{i L(s)},
$$

where

$$
L(s)=\int_{0}^{s} \kappa(u) d u
$$

is the total curvature function. Since $\gamma^{\prime}(s)=T(s)$, we integrate $\gamma^{\prime}(s)=$ $T(0) e^{i L(s)}$ to get

$$
\gamma(s)=\gamma(0)+T(0) \int_{0}^{s} e^{i L(u)} d u
$$

We may assume without loss of generality that $\gamma(0)=0$ and $T(0)=1$. Then

$$
\gamma(s)=\int_{0}^{s} \cos L(u) d u+i \int_{0}^{s} \sin L(u) d u .
$$


And the osculating circle is given by $|z-i|=1$. Now check $|\gamma(s)-i|^{2}$, namely

$$
\left(\int_{0}^{s} \cos L(u) d u\right)^{2}+\left(1-\int_{0}^{s} \sin L(u) d u\right)^{2} .
$$

Since $\kappa(u)>1$ on $(0, \delta)$, we have $L(u)>u$. Thus $\cos L(u)<\cos u$ and $\sin L(u)>\sin u$. Applying these to (1) for sufficiently small $s$, we have

$$
|\gamma(s)-i|<1 .
$$

Now assume $\kappa(0)=0$. Then we have only to show that

$$
\int_{0}^{s} \sin L(u) d u>0 .
$$

But it follows from the fact that $L(u)>0$.

The proof for the case $I=[-\delta, 0]$ is the same, except for a few minor modification: Define, for $s<0$,

$$
L(s)=\int_{s}^{0} \kappa(u) d u \text {. }
$$

Then the assumption implies $L(s)>-s$ when $\kappa(0)=1$, and $L(s)>0$ when $\kappa(0)=0$, and the relevant facts are

$$
T(s)=T(0) e^{-i L(s)},
$$

and

$$
\gamma(s)=\gamma(0)-T(0) \int_{s}^{0} e^{-i L(u)} d u
$$

But then the rest of the proof are essentially the same.

The proof for the case $\kappa(t)<\kappa(0)$ proceeds very similarly.

Remark 3.2. One can remove the assumption $\kappa(0) \geq 0$ in Lemma 3.1. When $\kappa(0)<0$, one can reverse the curve and use Lemma 3.1 to obtain the appropriate corresponding results.

The following theorem is about the basic geometric facts for osculating circles.

Theorem 3.1. Let $I$ be an interval of one of the types $[-\delta, \delta],[0, \delta]$ or $[-\delta, 0]$. Let $\gamma: I \rightarrow \mathbb{R}^{2}$ be a real analytic curve such that $\kappa(0) \geq 0$. Assume $\gamma$ lies outside (resp., inside) the osculating circle of $\gamma$ at $\gamma(0)$ in case $\kappa(0)>$ 0 . And in case $\kappa(0)=0$, assume $\gamma$ lies outside the half plane $P=\{p \in$ $\mathbb{R}^{2} \mid\langle\overrightarrow{\gamma(0) p}, N(0)\rangle>$ (resp., $\left.\left.<\right) 0\right\}$, where $N$ is the usual counterclockwise rotation of $\gamma^{\prime}$ by 90 degrees. Then $\kappa(0)$ is a local maximum (resp., minimum) of the curvature function. 
Proof. We first give a proof for the case when $\gamma$ lies outside the osculating circle or the half plane. We will give a proof for the case $I=[0, \delta]$, as the rest are the same. Suppose now that $\kappa(0)$ is not a local maximum. Then there exists a monotone decreasing sequence $u_{n} \downarrow 0$ such that $\kappa\left(u_{n}\right)>\kappa(0)$. If $\kappa(s)>\kappa(0)$ for all $s \in\left(0, u_{n}\right)$, then by Lemma 3.1, $\gamma(s)$ must lie strictly inside the osculating circle or the half plane. Thus there exists $s_{n} \in\left(0, u_{n}\right)$ such that $\kappa\left(s_{n}\right) \leq \kappa(0)$. Thus there exists $t_{n} \in\left[s_{n}, u_{n}\right]$ such that $\kappa\left(t_{n}\right)=$ $\kappa(0)$, and $t_{n} \downarrow 0$. Since $\kappa$ is a real analytic function which can be extended real analytically across 0 , it must be constant. Thus $\kappa(0)$ is a local maximum, which is a contradiction.

The rest of the proof proceeds similarly.

Remark 3.3. When we say $\gamma$ lies outside the osculating circle, we are allowing $\gamma$ to lie on the osculating circle. We do similarly for the half plane.

Remark 3.4. Let $\gamma(t)=\left(t,-e^{-1 / t^{2}} \sin ^{2} 1 / t\right)$. Then it is clearly a $C^{\infty}$ curve, and its curvature $\kappa(0)$ at $t=0$ is 0 . Note that it lies outside the half plane $P=\{(x, y) \mid y>0\}$. But there exists a sequence $t_{n} \rightarrow 0$ such that $\kappa\left(t_{n}\right)>0$. This example shows that the real analyticity assumption in Theorem 3.1 is an essential one. For the case $\kappa(0)>0$, one can certainly cook up similar examples.

Now let $\Omega$ be a domain. A circle in $\mathbb{R}^{2}$ is called an inscribed osculating circle in $\Omega$, if it is in $\Omega$ and is osculating at least one real analytic piece of $\partial \Omega$. Theorem 3.1 gives us the following finiteness result.

Corollary 3.1 (Finiteness of the Number of Inscribed Osculating Circles). The number of the inscribed osculating circles in a domain $\Omega$ is finite.

Proof. Suppose the number of the inscribed osculating circles is infinite. Since there are finite number of real analytic pieces in $\partial \Omega$, there is one real analytic piece, denoted by $\zeta$, which infinitely many distinct inscribed osculating circles osculate. Theorem 3.1 implies that $\zeta$ has an infinite number of points at which the curvature is local maximum. The curvature function of a real analytic curve is also real analytic, thus this implies that the curvature function must be constant. Namely, $\zeta$ must be a circular arc. But then it can have only one osculating circle, which is absurd.

\section{Medial Axis Transform.}

4.1. Definition of Medial Axis Transform. Let $\Omega$ be a domain in $\mathbb{R}^{2}$. Let $B_{r}(p)$ denote the closed disk of radius $r$ centered at $p$. We define the 
ordered set $\mathcal{D}(\Omega)$ by

$$
\mathcal{D}(\Omega)=\left\{B_{r}(p) \mid B_{r}(p) \subset \Omega\right\},
$$

which is ordered by the set inclusion. That is, $\mathcal{D}(\Omega)$ is the set of all closed disks contained in $\Omega$.

Remark 4.1. Throughout this paper, all disks are assumed to be closed disks unless stated otherwise. This assumption simplifies many arguments concerning closedness of objects in discussion. Furthermore, the radius of a disk is allowed to be 0 , in which case the disk is just a point.

Definition 4.1. The core of a domain $\Omega$ is the set of all maximal elements in $\mathcal{D}(\Omega)$, that is,

$$
\begin{aligned}
\operatorname{CORE}(\Omega)=\left\{B_{r}(p) \in \mathcal{D}(\Omega) \mid\right. & B_{s}(q) \in \mathcal{D}(\Omega) \\
& \text { and } \left.B_{r}(p) \subset B_{s}(q) \text { implies } B_{r}(p)=B_{s}(q)\right\} .
\end{aligned}
$$

We call $B_{r}(p)$ a maximal disk and $\partial B_{r}(p)$ a maximal circle, if $B_{r}(p) \in$ $\operatorname{CORE}(\Omega)$.

Now we define the medial axis and the medial axis transform.

Definition 4.2. The medial axis of a domain $\Omega$ is the set of the centers of disks in $\operatorname{CORE}(\Omega)$. That is,

$$
\mathbf{M A}(\Omega)=\left\{p \in \Omega \mid B_{r}(p) \in \mathbf{C O R E}(\Omega)\right\} .
$$

The medial axis transform of a domain $\Omega$ is the set of the ordered pairs of centers and radii of disks in $\operatorname{CORE}(\Omega)$. That is,

$$
\operatorname{MAT}(\Omega)=\left\{(p, r) \in \Omega \times\left(\mathbb{R}^{+} \cup\{0\}\right) \mid B_{r}(p) \in \operatorname{CORE}(\Omega)\right\} .
$$

Here the radius is allowed to be 0 of course. Such a case occurs at a sharp corner of $\partial \Omega$.

\subsection{Corner Points.}

Definition 4.3. A boundary point is a corner (point) if the unit tangent vector field is discontinuous at that point. It is called a sharp (resp., dull) corner if the interior angle is strictly less (resp., greater) than $\pi$.

Definition 4.4. Let $\gamma(t)$ be a piecewise real analytic curve which is a part of the boundary of $\Omega$. We assume that $\gamma$ is oriented in such a way that the interior of $\Omega$ is always on the left of $\gamma$. Let $p=\gamma(0)$ and suppose $p$ is not a sharp corner. We define the inward unit cone $I C(p)$ at $p$ by

$$
\begin{aligned}
I C(p)=\{\vec{v}:|\vec{v}|= & 1, \vec{v} \text { is an inward pointing vector at } p \\
& \text { such that } \left.\left\langle\vec{v}, \gamma^{\prime}(0+)\right\rangle \leq 0 \text { and }\left\langle\vec{v}, \gamma^{\prime}(0-)\right\rangle \geq 0\right\} .
\end{aligned}
$$


Thus if $\gamma$ is differentiable at $p, I C(p)$ consists of the single inward unit normal vector.

We say $\vec{w} \in I C(p)$ is an interior direction if $\left\langle\vec{w}, \gamma^{\prime}(0+)\right\rangle \leq 0$ and $\left\langle\vec{w}, \gamma^{\prime}(0-)\right\rangle \geq 0$, and a purely interior direction if $\left\langle\vec{w}, \gamma^{\prime}(0+)\right\rangle<0$ and $\left\langle\vec{w}, \gamma^{\prime}(0-)\right\rangle>0$ (i.e., $\vec{w}$ is in the interior of $I C(p)$ ).

4.3. Contact Points. From now on, for a medial axis point $p$, let $B(p)$ denote the disk $B_{r}(p)$ in $\mathbf{C O R E}(\Omega)$ which corresponds to $p$. The distance $d(p, \partial \Omega)$ is realized at the contact points of $\partial B(p)$ with $\partial \Omega$. That is, $d(p, \partial \Omega)=d(p, q)$ for any $q \in \partial \Omega \cap \partial B(p)$. If the boundary $\partial \Omega$ has a circular arc portion whose corresponding circle is contained in $\Omega$, and $p$ is the center of this circle, then $p$ is in $\mathbf{M A}(\Omega)$ and the distance $d(p, \partial \Omega)$ is realized at every point in the circular arc portion. Thus the distance can be realized at infinitely many boundary points. So the important information is not the number of points where the distance is realized but the number of connected components of the set of all common points between $B(p)$ and $\partial \Omega$. In this regard, we give the following definition.

Definition 4.5. Let $B(p)$ be a disk in $\operatorname{CORE}(\Omega)$. Then we define the contact set of $p$ (or of $B(p)$, or of $\partial B(p)$ ), denoted by $C(p)$, as

$$
C(p)=\partial B(p) \cap \partial \Omega .
$$

A point in $C(p)$ is called a contact point of $p$ (or of $B(p)$, or of $\partial B(p)$ ). A connected component of $C(p)$ is called a contact component of $p$ (or of $B(p)$, or of $\partial B(p))$, and a contact component is called an isolated contact point if it is a point, and a contact arc if it is an arc containing its two end points. Finally, $\partial B(p)$ is called a contact circle.

It is trivial to see that a connected set in the real line is either a point or an interval, thus a contact component is either an isolated contact point or a contact arc.

Lemma 4.1 (Finiteness of the Number of Contact Components). Let $B_{r}(p)$ be a disk in $\mathbf{C O R E}(\Omega)$. Then the number of the contact components of $p$ is finite.

Proof. Suppose that $p$ has infinitely many contact components. Then there is a real analytic piece $\gamma:[a, b] \rightarrow \mathbb{R}^{2}$ of $\partial \Omega$ which have infinitely many contact components of $p$. Define the function $\rho:[a, b] \rightarrow \mathbb{R}$ by $\rho(t)=d(p, \gamma(t))$. Since $\rho$ is a real analytic function defined on a closed interval which has infinitely many local minima, $\rho$ must be constant. But then, this means that $\gamma$ is a part of a circular arc of $B(p)$, which is absurd. 
In fact, almost all medial axis points have two contact components, which will be clear in Section 6.3. However some medial axis points can have only one contact component or more than two contact components, and we characterize the medial axis points by the number of their contact components.

Definition 4.6. A point $p$ in $\mathbf{M A}(\Omega)$, which is not a sharp corner point, is called an $n$-prong (point) where $n \geq 1$, if $C(p)$ has $n$ contact components. For reasons that will become clear when we deal with the graph structure of the medial axis, we classify the sharp corner points as 1-prong points.

Definition 4.7. An $n$-prong point $p$ for $n \geq 3$ is called a bifurcation point, and a 1-prong point is also called a terminal point. Let $(p, r)$ be in $\operatorname{MAT}(\Omega)$. The disk $B_{r}(p) \in \mathbf{C O R E}(\Omega)$ is called a bifurcation disk, if $p$ is a bifurcation point. In this case, $\partial B_{r}(p)$ is called a bifurcation circle. The disk $B_{r}(p) \in \mathbf{C O R E}(\Omega)$ is called an osculating disk (at $\left.q \in \partial \Omega\right)$, if $\partial B(p)$ is an inscribed circle which osculates $\partial \Omega$ (at $q)$.

The following lemma is useful in dealing with the terminal points.

Lemma 4.2. A 1-prong point, which is not a sharp corner, is the center of an inscribed osculating circle with one contact component.

Proof. Let $p$ be a 1-prong point which is not a sharp corner, and let $r>0$ be the radius of the corresponding 1-prong circle with the center $p$. There are two possibilities for the contact component: Either it is a circular arc or a single point. If it is a circular arc, the proof is obvious. So assume that the contact component consists of a single point $q$. Let $\kappa^{+}(q)$ and $\kappa^{-}(q)$ be defined as above. First, note that $\max \left\{\kappa^{+}(q), \kappa^{-}(q)\right\}>0$. Now, clearly $1 / r \geq \max \left\{\kappa^{+}(q), \kappa^{-}(q)\right\}$. Suppose $1 / r>\max \left\{\kappa^{+}(q), \kappa^{-}(q)\right\}$. By rotating the domain, we may assume without loss of generality that $q=0$ and the $x$-axis is tangent to $\partial \Omega$. Then $\partial \Omega$ can be described locally near $q$ as the graph of a function

$$
f(x)=f(0)+f^{\prime}(0) x+\frac{k^{+}(q)}{2} x^{2}+O\left(|x|^{3}\right) \quad \text { for } x \geq 0,
$$

and

$$
f(x)=f(0)+f^{\prime}(0) x+\frac{k^{-}(q)}{2} x^{2}+O\left(|x|^{3}\right) \text { for } x \leq 0,
$$

while the circle of radius $r$ with the center $p$ is represented as the graph of a function

$$
g(x)=g(0)+g^{\prime}(0) x+\frac{g^{\prime \prime}(0)}{2} x^{2}+O\left(|x|^{3}\right) .
$$


Enlarge $B_{r}(p)$ to $B_{r+\epsilon}\left(p_{\epsilon}\right)$ by moving the center $p$ to $p_{\epsilon}$ away from $q$ while still staying on the line from $q$ to $p$ to make $d\left(p_{\epsilon}, q\right)=r+\epsilon$. Since $f(0)=g(0)=0$, $f^{\prime}(0)=g^{\prime}(0)=0$, and $1 / r=g^{\prime \prime}(0)>\max \left\{\kappa^{+}(q), \kappa^{-}(q)\right\}$, one can find sufficiently small $\epsilon>0$ and $\delta>0$ such that $B_{r+\epsilon}\left(p_{\epsilon}\right) \cap B_{\delta}(q)$ still stays inside $\Omega$. Now outside $B_{\delta}(q)$, the boundary $\partial \Omega$ and the boundary of $B_{r}(p)$ has some positive distance, so for sufficiently small $\epsilon, B_{r+\epsilon}\left(p_{\epsilon}\right)$ and $\partial \Omega$ do not meet outside $B_{\delta}(q)$ either. Therefore, $B_{r}(p)$ cannot be a maximal disk. Hence, $1 / r$ must be equal to $\max \left\{\kappa^{+}(q), \kappa^{-}(q)\right\}$.

By combining the above lemma and Corollary 3.1, we have the following.

Corollary 4.1 (Finiteness of the Number of 1-prongs). The number of 1-prongs in a domain is finite.

\section{Domain Decomposition Lemma.}

In this section, we introduce our fundamental tool, called the Domain Decomposition Lemma. By this lemma, we can decompose the given domain into many small subdomains. And the medial axis (resp., medial axis transform) of the domain is represented by the union of all subdomains' medial axes (resp., medial axis transforms). See also [6] for related results and discussions. The basic idea of the domain decomposition is illustrated in Figure 3.
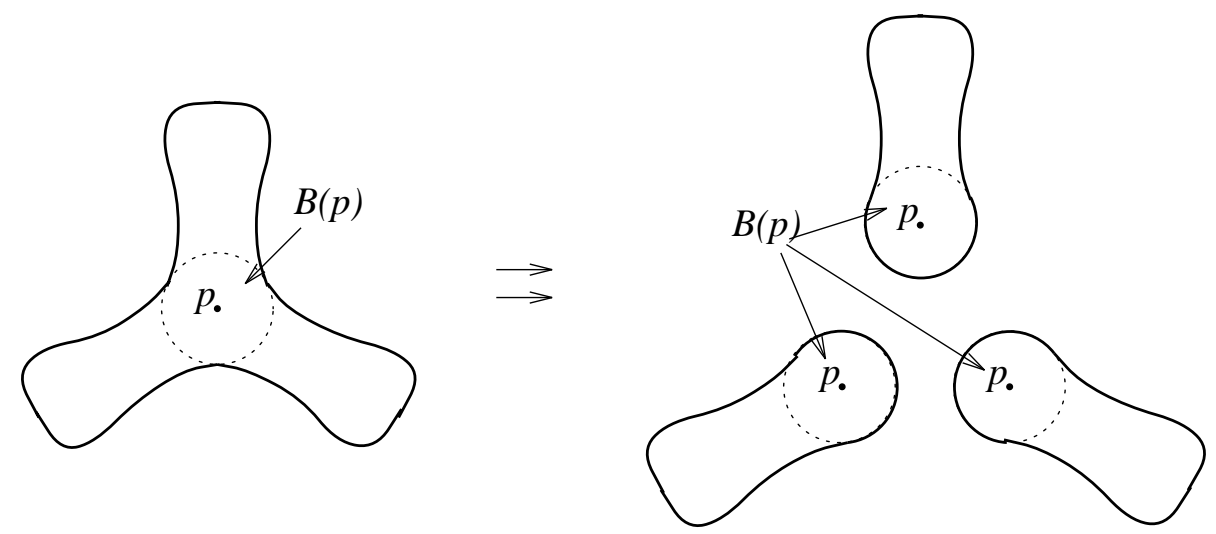

Figure 3. The Idea of Domain Decomposition.

For a given domain $\Omega$, let $p$ be a medial axis point and $B(p)$ be the corresponding maximal disk in $\operatorname{CORE}(\Omega)$. Now let $A_{1}, \ldots, A_{n}$ be the connected 
components of $\Omega \backslash B(p)$, and we define the subset $T(p)$ of $B(p)$ to be the union of all segments from $p$ to contact points of $B(p)$ with $\partial \Omega$. For $i=1,2, \ldots, n$, let $B_{i}$ be the union of the connected components of $B(p) \backslash T(p)$ which border with $A_{i}$. Then $B(p) \backslash T(p)=\cup_{i=1}^{n} B_{i}$.

Now we have some basic facts about $T(p)$ and $\mathcal{D}(\Omega)$.

Lemma 5.1. $T(p) \cap \mathbf{M A}(\Omega)=\{p\}$.

Proof. Suppose $q \in T(p) \cap \mathbf{M A}(\Omega)$. If $q \neq p$, then $q$ lies on a line segment from $p$ to a contact point $p^{\prime}$ on $\partial B(p)$. Let $r=d(q, \partial \Omega)=d\left(q, p^{\prime}\right)$. As $B_{r}(q)$ is properly contained in $B(p), B_{r}(q)$ cannot be a maximal disk. Thus $q \notin \mathbf{M A}(\Omega)$, and therefore $p=q$.

Lemma 5.2. $\mathcal{D}(\Omega)=\bigcup_{i=1}^{n} \mathcal{D}\left(A_{i} \cup B(p)\right)$.

Proof. Suppose $B_{r}(q)$ is any disk in $\mathcal{D}(\Omega)$. As we have seen before, if $q \in T(p)$ then $B_{r}(q) \subset B(p)$. Thus $B_{r}(q) \in \bigcup_{i=1}^{n} \mathcal{D}\left(A_{i} \cup B(p)\right)$. If $q \notin T(p)$ then $q \in A_{i} \cup B_{i}$ for some $i$. We want to show that $B_{r}(q) \subset A_{i} \cup B(p)$. Suppose $B_{r}(q) \not \subset A_{i} \cup B(p)$. Then there exists $q^{\prime}$ in $A_{j}$ for some $j \neq i$ such that $d\left(q, q^{\prime}\right)=r$. Note that the line segment from $q$ to $q^{\prime}$ should go through a point $w$ in $T(p)$. Let $p^{\prime}$ be the boundary point such that $w \in \overline{p p^{\prime}}$. Therefore we get $r=d(q, \partial \Omega) \leq d\left(q, p^{\prime}\right) \leq d(q, w)+d\left(w, p^{\prime}\right)$. Since $q^{\prime} \notin B(p)$, we have $d\left(w, p^{\prime}\right)<d\left(w, q^{\prime}\right)$. So $r<d(q, w)+d\left(w, q^{\prime}\right)=d\left(q, q^{\prime}\right)=r$, which is a contradiction.

Lemma 5.3. For $i \neq j, \mathcal{D}\left(A_{i} \cup B(p)\right) \cap \mathcal{D}\left(A_{j} \cup B(p)\right)=\mathcal{D}(B(p))$.

Proof. Suppose $B_{r}(q)$ is a disk in $\mathcal{D}\left(A_{i} \cup B(p)\right) \cap \mathcal{D}\left(A_{j} \cup B(p)\right)$. If $q \in T(p)$, then the proof is trivial as before. Suppose $q \notin T(p)$. Then we can assume with no loss of generality that $q \in A_{i} \cup B_{i}$, and as we have seen before, $B_{r}(q)$ cannot meet any point in $A_{j}$. That is, $B_{r}(q) \cap A_{j}=\emptyset$. Since $B_{r}(q) \subset$ $A_{j} \cup B(p)$, we must have $B_{r}(q) \subset B(p)$.

Theorem 5.1 (Domain Decomposition Lemma). For any fixed medial axis point $p \in \mathbf{M A}(\Omega)$, let $B(p)\left(=B_{r}(p)\right)$ be the corresponding maximal disk, i.e., $B(p) \in \mathbf{C O R E}(\Omega)$. Suppose $A_{1}, \ldots, A_{n}$ are the connected components of $\Omega \backslash B(p)$. Denote $\Omega_{i}=A_{i} \cup B(p)$ for $i=1, \ldots, n$. Then

$$
\mathbf{M A}(\Omega)=\bigcup_{i=1}^{n} \mathbf{M A}\left(\Omega_{i}\right)
$$

and

$$
\operatorname{MAT}(\Omega)=\bigcup_{i=1}^{n} \operatorname{MAT}\left(\Omega_{i}\right)
$$


Moreover, we have

$$
\mathbf{M A}\left(\Omega_{i}\right) \cap \mathbf{M A}\left(\Omega_{j}\right)=\{p\}
$$

and

$$
\operatorname{MAT}\left(\Omega_{i}\right) \cap \operatorname{MAT}\left(\Omega_{j}\right)=\{(p, r)\},
$$

for every distinct $i$ and $j$.

Proof. Suppose $(q, s) \in \operatorname{MAT}\left(\Omega_{i}\right)$ for some $i$. We will show that $B_{s}(q)$ is also maximal disk in $\Omega$. If there exists another disk $B_{s^{\prime}}\left(q^{\prime}\right) \in \mathcal{D}(\Omega)$ such that $B_{s}(q) \subset B_{s^{\prime}}\left(q^{\prime}\right)$, then by Lemma 5.2, $B_{s^{\prime}}\left(q^{\prime}\right) \in \mathcal{D}\left(\Omega_{j}\right)$ for some $j$. If $i=j$, then $B_{s}(q)=B_{s^{\prime}}\left(q^{\prime}\right)$ by the maximality of $B_{s}(q)$ in $\mathcal{D}\left(\Omega_{i}\right)$. If $i \neq j$, then we get $B_{s}(q) \subset B_{s^{\prime}}\left(q^{\prime}\right) \in \mathcal{D}\left(\Omega_{j}\right)$. So $B_{s}(q) \in \mathcal{D}\left(\Omega_{i}\right) \cap \mathcal{D}\left(\Omega_{j}\right)=\mathcal{D}(B(p))$ by Lemma 5.3. Since $B_{s}(q)$ is maximal in $\mathcal{D}\left(\Omega_{i}\right)$, it is also maximal in $\mathcal{D}(B(p))$, which means that $B_{s}(q)=B(p)$. In either case, $B_{s}(q)$ is maximal in $\mathcal{D}(\Omega)$. So $(q, s) \in \operatorname{MAT}(\Omega)$. This proves $\operatorname{MAT}(\Omega) \supset \cup_{i=1}^{n} \operatorname{MAT}\left(\Omega_{i}\right)$.

On the other hand, suppose $(q, s) \in \mathbf{M A T}(\Omega)$. Then by Lemma 5.2, $B_{s}(q) \in \mathcal{D}\left(\Omega_{i}\right)$ for some $i$. Since $B_{s}(q)$ is maximal in $\mathcal{D}(\Omega), B_{s}(q)$ is also maximal in $\mathcal{D}\left(\Omega_{i}\right)$. That is, $(q, s) \in \operatorname{MAT}\left(\Omega_{i}\right)$. Thus we proved $\operatorname{MAT}(\Omega) \subset$ $\bigcup_{i=1}^{n} \operatorname{MAT}\left(\Omega_{i}\right)$.

Suppose $(q, s) \in \operatorname{MAT}\left(\Omega_{i}\right) \cap \operatorname{MAT}\left(\Omega_{j}\right)$. Then $B_{s}(q) \in \mathcal{D}\left(\Omega_{i}\right) \cap \mathcal{D}\left(\Omega_{j}\right)$. Thus, by Lemma $5.3, B_{s}(q) \in \mathcal{D}(B(p))$. Since $B_{s}(q)$ is maximal in $\mathcal{D}(\Omega)$, it is also maximal in $\mathcal{D}(B(p))$, and hence $B_{s}(q)=B(p)$. This proves $\operatorname{MAT}\left(\Omega_{i}\right) \cap$ $\operatorname{MAT}\left(\Omega_{j}\right)=\{(p, r)\}$ for every $i \neq j$.

The results for $\mathbf{M A}(\Omega)$ follows immediately from the above.

Remark 5.1. The Domain Decomposition Lemma can be used to localize the arguments about $\mathbf{M A T}(\Omega)(\mathbf{M A}(\Omega))$ : decompose the domain $\Omega$ recursively by using the maximal disks $B_{r_{1}}\left(p_{1}\right), \ldots, B_{r_{n}}\left(p_{n}\right)$ in $\operatorname{CORE}(\Omega)$. Let $\Omega_{1}, \ldots, \Omega_{N}$ be the resulting subdomains. Then, by using the above domain decomposition lemma, we can easily see that $\mathbf{M A T}(\Omega)=\cup_{i=1}^{N} \mathbf{M A T}\left(\Omega_{i}\right)$, and $\mathbf{M A T}\left(\Omega_{i}\right) \cap \mathbf{M A T}\left(\Omega_{j}\right)=\left(p_{k}, r_{k}\right)$ for some $k(1 \leq k \leq n)$ and for every $i \neq j$. Of course, the same statement can be made for $\mathbf{M A}(\Omega)$. In short, the global information about the medial axis (transform) can be obtained by combining local informations about the medial axis (transform) only, and local informations are independent of the global information.

\section{Basic Notations and Lemmas.}

6.1. Extended Boundary. We first make the following observation. Let $q$ be a point in $\partial \Omega$. If $q$ is not a corner, then there exists a unique point $p$ in 
$\mathbf{M A}(\Omega)$ such that $q \in C(p)$. Moreover, $\overrightarrow{q p}$ is in the inner normal direction at $q$. Let $q$ be a dull corner. Then, for each interior direction of $q$, there exists a unique point $p$ in $\mathbf{M A}(\Omega)$ such that $q \in C(p)$. For a direction not in $I C(q)$, there are no points $p$ in $\mathbf{M A}(\Omega)$ such that $q \in C(p)$. Finally, if $q$ is a sharp corner, then $q$ is the only point in $\mathbf{M A}(\Omega)$ such that $q \in C(p)$.

In our analysis, we need to analyze the correspondence between the points in $\partial \Omega$ and the points in $\mathbf{M A}(\Omega)$ (or $\mathbf{M A T}(\Omega)$ ), and the above observation can be used to establish the correspondence. But there are some problems for dull corners, for, as we have observed, there corresponds a point $p$ in $\mathbf{M A}(\Omega)$ (and $(p, r) \in \mathbf{M A T}(\Omega))$ for each interior direction of a dull corner. This prevents us to define a map from $\partial \Omega$ to $\mathbf{M A}(\Omega)(\mathbf{M A T}(\Omega))$, and thus we need some concept generalizing the real boundary $\partial \Omega$, which we call the extended boundary.

Let $\Omega$ be a domain with genus $g$, and let $C_{1}, C_{2}, \ldots, C_{g+1}$ be the simple closed curves in $\partial \Omega$. Then by our standing assumption on the domain, there exists a continuous map $\tilde{\gamma}_{i}:[0,1] \rightarrow C_{i}$ such that $\tilde{\gamma}_{i}(0)=\tilde{\gamma}_{i}(1)$ for each $i$. We slightly modify this map as follows. For each dull corner in $C_{i}$, we replace the corresponding point in $[0,1]$ by a closed interval which parametrize continuously the interior directions of that dull corner. Each interior direction which corresponds to an end point of this inserted interval should be the limit of the inner normal directions at points in $\partial \Omega$, as the corresponding parameters of these points approach to that end of this interval from outside. By resizing the resulting intervals, we can redefine a map $\gamma_{i}:[0,1] \rightarrow\left(C_{i} \backslash\left\{\right.\right.$ dull corners in $\left.\left.C_{i}\right\}\right) \cup \bigcup_{q \text { : dull corners in } C_{i}} I C(q)$ such that $\gamma_{i}(0)=\gamma_{i}(1)$ for each $i$.

Let $S^{1}$ be the unit circle, and let $T_{1}, \ldots, T_{g+1}$ be disjoint copies of $S^{1}$. Now let $\partial \Omega^{*}$ be $\cup_{i=1}^{g+1} T_{i}$, where each $T_{i}$ is viewed as the domain $[0,1]$ of the map $\gamma_{i}$ with 0 and 1 identified. In short, we have parametrized the set $(\partial \Omega \backslash\{$ dull corners $\}) \cup \bigcup_{q}$ : dull corner $I C(q)$ by the set $\partial \Omega^{*}=\cup_{i=1}^{g+1} T_{i}$. In what follows, we will always assume that one such parametrization is given.

Definition 6.1 (Extended Boundary). We call $\partial \Omega^{*}$ the extended boundary of $\Omega$ assuming the above parametrization is fixed.

Remark 6.1. We will often identify an element in $\partial \Omega^{*}$ with the corresponding one in the set $(\partial \Omega \backslash\{$ dull corners $\}) \cup \bigcup_{q}$ : dull corner $I C(q)$. In some situations, we will also identify the points in $\partial \Omega^{*}$ with real numbers, as it is convenient if we can refer an arc in $\partial \Omega^{*}$ as an interval of real numbers. These identifications enable us to simplify our notations when we need to deal with the boundary points which are not dull corners and the interior directions of the dull corners at the same time. 
By virtue of the above definition of extended boundary, now we can give the following definition.

Definition 6.2 (Medial Axis (Transform) Map along the Boundary). The medial axis transform map (along the extended boundary), $\tilde{\beta}$ : $\partial \Omega^{*} \rightarrow \mathbf{M A T}(\Omega)$, given by $\tilde{\beta}(t)=(p(t), r(t))$ is defined as follows.

(1) If $t \in \partial \Omega^{*}$ corresponds to a point $q$ in $\partial \Omega$ which is not a dull corner, then $(p(t), r(t))$ is the unique point in $\operatorname{MAT}(\Omega)$ such that $q \in C(p(t))$.

(2) If $t \in \partial \Omega^{*}$ corresponds to an interior direction $\vec{v}$ of a dull corner $q$, then $(p(t), r(t))$ is the unique point in $\mathbf{M A T}(\Omega)$ such that $p(t)$ is in the half line from $q$ in the direction of $\vec{v}$, and $q \in C(p(t))$.

We define the map $\beta: \partial \Omega^{*} \rightarrow \mathbf{M A}(\Omega)$, which is called the medial axis map (along the extended boundary), by $\beta=\pi \circ \tilde{\beta}$, where $\pi$ is the projection $\operatorname{map} \pi: \mathbf{M A T}(\Omega) \rightarrow \mathbf{M A}(\Omega)$, given by $\pi((p, r))=p$.

We will prove that the maps $\tilde{\beta}$ and $\beta$ are continuous in Section 7.2.

6.2. Finiteness of the Number of Bifurcation Points. In this section, we prove an important finiteness result concerning the number of the bifurcation points. First, we need the following lemma.

Lemma 6.1. $\quad$ Let $\Omega$ be a simply connected domain without any corner. Then there exist at least two inscribed osculating circles.

Proof. Take any $B(p) \in \mathbf{C O R E}(\Omega)$. If $\partial B(p)$ is an inscribed osculating circle with only one contact component, let $\Omega_{1}$ be the whole domain $\Omega$. We will show that $\Omega_{1}$ has another inscribed osculating circle, which completes the proof. If $B(p)$ decomposes $\Omega$ into more than one subdomains, then take any one of them, and call it $\Omega_{1}$. We will also show that $\Omega_{1}$ has an inscribed osculating circle other than $\partial B(p)$. Then the proof will be complete, since there is another such subdomain.

Now let $\Omega_{1}$ be either one of those chosen above. It is easy to see that $\Omega_{1}$ is simply connected. Thus the boundary of $\Omega_{1}$ consists of two portions: one is a circular arc portion (or a point) which is $\partial \Omega_{1} \cap B(p)$, and the other is a curve, called $\gamma_{0}$, defined to be $\partial \Omega_{1} \backslash B(p)$. Take a point $q_{1}$, in $\gamma_{0}$ which is at half length of $\gamma_{0}$, and take the maximal disk $B\left(p_{1}\right) \in \mathbf{C O R E}\left(\Omega_{1}\right)$ which makes contact with $\Omega_{1}$ at $q_{1}$. If $B\left(p_{1}\right)$ has a contact arc, or has only one contact component with $\partial \Omega_{1}$, then we are done by Lemma 4.2. Otherwise, continue as follows: First note that $B\left(p_{1}\right)$ has no contact with $\partial \Omega_{1} \cap B(p)$. For, otherwise, $B\left(p_{1}\right) \subset B(p) \subset \Omega$, hence $B\left(p_{1}\right)=B(p)$, since $B\left(p_{1}\right) \in \mathbf{C O R E}\left(\Omega_{1}\right)$ implies $B\left(p_{1}\right) \in \mathbf{C O R E}(\Omega)$. But this is absurd. Thus $B\left(p_{1}\right)$ has at least two isolated contact points with $\partial \Omega_{1}$, none of which lies 
in $\partial \Omega_{1} \cap B(p)$. Decompose $\Omega_{1}$ with $B\left(p_{1}\right)$. Then there is a subdomain $\Omega_{2}$ containing $q_{1}$ which is disjoint from $\partial \Omega_{1} \cap B(p)$. It is easy to see that there is a unique $q_{1}^{*}$ in $\partial B\left(p_{1}\right) \cap \Omega_{2}$ such that $q_{1}^{*} \neq q_{1}$ and the portion of $\gamma_{0}$ between $q_{1}$ and $q_{1}^{*}$ has no other points in $\partial B\left(p_{1}\right)$. Call this portion $\gamma_{1}$. This process is illustrated in Figure 4.

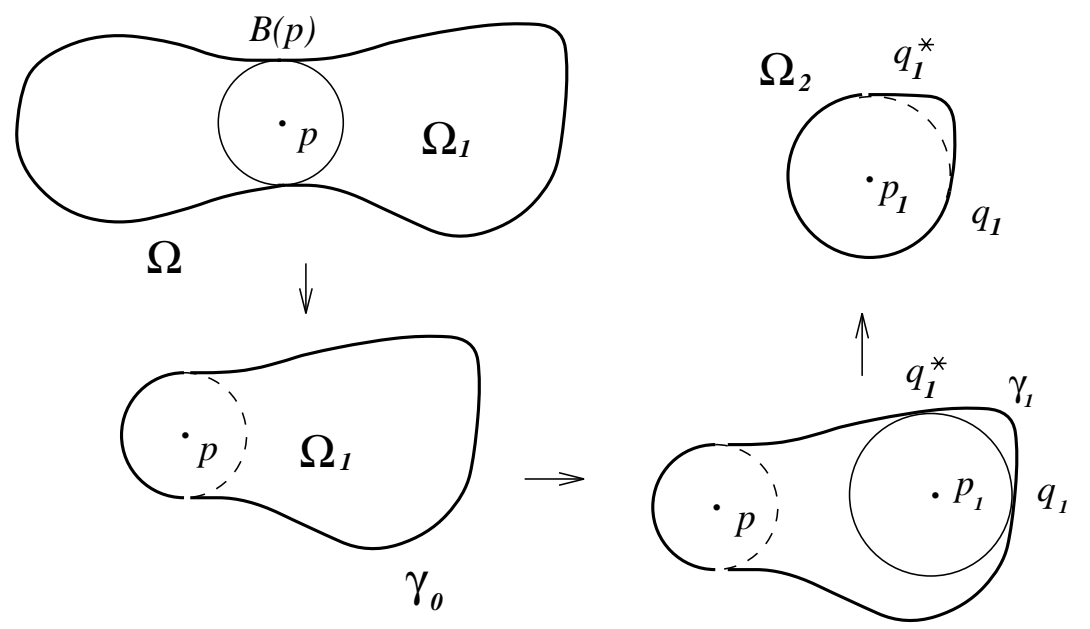

Figure 4. Successive Subdomains.

Note that $\gamma_{1}$ has the length less than or equal to the half of the length of $\gamma_{0}$. Now apply this process again to the subdomain $\Omega_{2}$ as a new domain. If we continue the above processes recursively, then either this process stops at a finite step in which case we are done, or this process continues indefinitely. In the latter case, we can find a sequence $\gamma_{n}$ such that $\gamma_{n}$ is a portion of $\gamma_{n-1}$ and the arc length of $\gamma_{n}$ is less than or equal to that of $\gamma_{n-1}$, where $\gamma_{0}$ is as given at the beginning. Because the length decreases at least by half at each step, $\gamma_{n}$ converges to a point, which we call $q^{*}$.

Now there exists a unique maximal disk $B\left(p^{*}\right)$ which has contact with $\partial \Omega_{1}$ at $q^{*}$. We claim that $q^{*}$ is the unique contact point of $p^{*}$. Suppose there is another point $q^{\prime} \in \partial \Omega_{1} \cap \partial B\left(p^{*}\right)$. Since $\gamma_{n}$ converges to $q^{*}, \gamma_{n}$ does not contain $q^{\prime}$ for sufficiently large $n$. But by applying the domain decomposition lemma, $B\left(p^{*}\right) \in \mathbf{C O R E}\left(\Omega_{n+1}\right)$. This is a contradiction, since $q^{\prime} \notin \Omega_{n+1}$. Thus $q^{*}$ is the only contact point of $p^{*}$. Now the proof follows by Lemma 4.2. 
Remark 6.2. By modifying the above proof slightly, one can in fact show that $\Omega$ has at least two inscribed osculating circles with only one contact component. In case there is no sharp corners, but dull corners are allowed, then we can still have the same conclusion with slight modification of the proof.

Remark 6.3. For a general simply connected domain with sharp corners and dull corners, the statement in Lemma 6.1 is still true, if the sharp corners are regarded as the inscribed osculating circle with zero radius, which implies that there exist at least two 1-prongs.

Theorem 6.1 (Finiteness of the Number of Bifurcation Points). The number of the bifurcation points in $\mathbf{M A}(\Omega)$ is finite.

Proof. Suppose there are infinite number of bifurcation points $p_{n} \in \mathbf{M A}(\Omega)$ $(n=1,2, \ldots)$. Let $B_{r_{n}}\left(p_{n}\right)(n=1,2, \ldots)$ be the corresponding disks in $\operatorname{CORE}(\Omega)$. Then, by the compactness of $\Omega$, one can choose appropriate subsequences, still called $\left\{p_{n}\right\}$ and $\left\{r_{n}\right\}$, of $\left\{p_{n}\right\}$ and $\left\{r_{n}\right\}$ such that $p_{n} \rightarrow$ $p_{0} \in \Omega$ and $r_{n} \rightarrow r_{0}$ for some $p_{0}$ and $r_{0}$. Then it is easy to see that $B_{r_{0}}\left(p_{0}\right) \in$ $\mathcal{D}(\Omega)$. Choose some $B_{\rho}(q) \in \mathbf{C O R E}(\Omega)$ such that $B_{\rho}(q) \supset B_{r_{0}}\left(p_{0}\right)$. Then for sufficiently small $\epsilon>0,\left(B_{\rho+\epsilon}(q) \cap \Omega\right) \backslash B_{\rho}(q)$ consists of $m$ disjoint sets $A_{1}, \ldots, A_{m}$, where $m$ is the number of the contact components of $q$. For each $i, A_{i} \cap \partial \Omega$ consists of two curves $\alpha_{i}$ and $\beta_{i}$. Note that the curves $\alpha_{i}$ and $\beta_{i}$, for $i=1, \ldots, m$, are all mutually disjoint, and we let $a_{i}=\overline{\alpha_{i}}$ and $b_{i}=\overline{\beta_{i}}$ for $i=1, \ldots, m$.

By the real analyticity, we may choose $\epsilon>0$ sufficiently small so that each $\alpha_{i}$ or $\beta_{i}$ for $i=1, \ldots, m$ is real analytic, and has no corners and no local maximum points for its curvature function: corners or curvature local maximum points may occur only at points in $a_{i} \cap B_{\rho}(q)$ or $b_{i} \cap B_{\rho}(q)$ for some $i=1, \ldots, m$. Since $B_{r_{n}}\left(p_{n}\right)$ converges to $B_{r_{0}}\left(p_{0}\right) \subset B_{\rho}(q), B_{r_{n}}\left(p_{n}\right) \subset$ $B_{\rho+\epsilon}(q)$ for all sufficiently large $n$. It is easy to see, by using the argument in the proof of Lemma 5.2, that once $B_{r_{n}}\left(p_{n}\right)$ makes contact with $a_{j}$ or $b_{j}$, then it cannot make contact with any other $a_{k}$ or $b_{k}$ for $k \neq j$. Since $p_{n}$ is a bifurcation point, $B_{r_{n}}\left(p_{n}\right)$ must make contact at least twice with one of $a_{j}$ and $b_{j}$. We may assume without loss of generality that there is a subsequence of $B_{r_{n}}\left(p_{n}\right)$, still denoted by $B_{r_{n}}\left(p_{n}\right)$, each of which makes contact with $a_{1}$ at least twice. See Figure 5. 


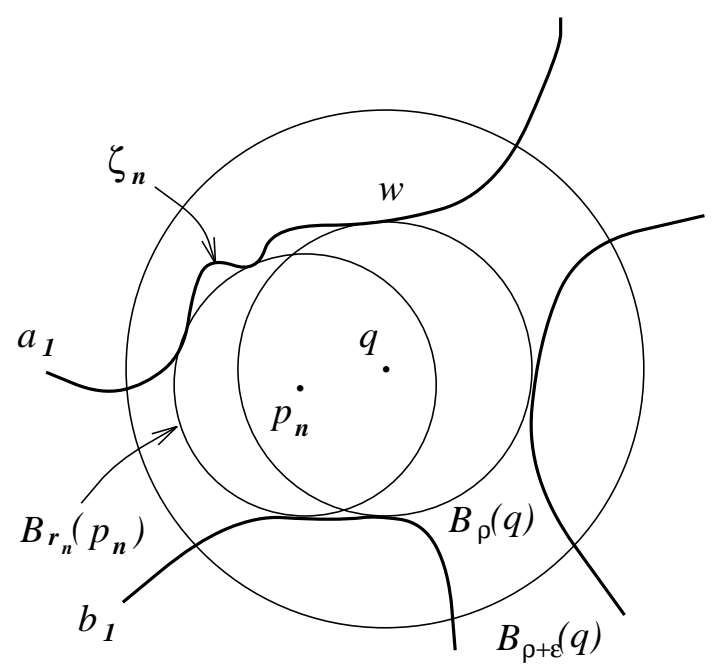

Figure 5. Limit of Bifurcation Circles.

Let $w$ be the unique point in $a_{1} \cap B_{\rho}(q)$. Now by applying the domain decomposition with $B_{r_{n}}\left(p_{n}\right)$, we can find a subdomain $\Omega_{n}$ whose boundary contains the portion $\zeta_{n}$ of $a_{1}$ which connects a pair of contact points of $p_{n}$. If, for some $n$, none of these contact points is $w$, then $\Omega_{n}$ has no corners, thus by Lemma $6.1, \zeta_{n}$ has a local maximum point for its curvature function, which contradicts our choice of $\epsilon$. Thus we may assume that for every sufficiently large $n$, one of these contact points is $w$. Call the other contact point $w_{n}$. (Note that this situation may happen only when $w$ is a dull corner of $\Omega$.) Since $B_{r_{n}}\left(p_{n}\right)$ converges to $B_{r_{0}}\left(p_{0}\right) \subset B_{\rho}(q), w_{n}$ must converge to $w$. Fix sufficiently large $n_{1}$. Then there is $n_{2}$ such that $w_{n_{2}}$ lies on $a_{1}$ between $w_{n_{1}}$ and $w$. Now by Domain Decomposition Lemma using $B_{r_{n_{1}}}\left(p_{n_{1}}\right)$, we can see easily that $B_{r_{n_{2}}}\left(p_{n_{2}}\right)$ has no contact with $b_{1}$. Thus $B_{r_{n_{2}}}\left(p_{n_{2}}\right)$ must have another contact point with $a_{1}$ other than $w_{n_{2}}$ and $w$. Call it $w^{\prime}$. Then by applying Lemma 6.1 , we can see that there exists a point in $a_{1}$ between $w^{\prime}$ and $w_{n_{2}}$ where the curvature takes a local maximum, which is impossible for our choice of $\epsilon$. Thus we have the desired result.

6.3. Generic 2-prong. In this section, we define some useful concepts: generic 2-prong and generic points in $\partial \Omega^{*}$.

Definition 6.3 (Generic 2-prong). A 2-prong $p$ in $\mathbf{M A}(\Omega)$ is a generic 2-prong, if the following conditions are satisfied.

(1) The two contact components of $p$ are isolated contact points (denoted by $q_{1}$ and $q_{2}$ ). 
(2) If $q_{i}(i=1,2)$ is not a dull corner, then $\partial \Omega$ near $q_{i}$ is real analytic, and $p$ is within the focal locus of a small piece of $\partial \Omega$ near $q_{i}$.

(3) If $q_{i}(i=1,2)$ is a dull corner, then $\overrightarrow{q_{i} p}$ is in a purely interior direction of $q_{i}$.

The next theorem justifies the term 'generic', and this follows immediately from the finiteness results such as Corollary 4.1, Theorem 6.1, and Corollary 3.1, all of which need the real analyticity of $\partial \Omega$.

Theorem 6.2 (Genericity of Generic 2-prongs). The number of points in $\mathbf{M A}(\Omega)$ which are not generic 2-prong is finite.

We define one more concept which is a companion to that of generic 2prong.

Definition 6.4. A point $t$ in $\partial \Omega^{*}$ is called generic, if $\beta(t) \in \mathbf{M A}(\Omega)$ is a generic 2-prong, where $\beta: \partial \Omega^{*} \rightarrow \mathbf{M A}(\Omega)$ is the medial axis map defined in Section 6.1.

\subsection{Boundary Generation from Medial Axis Transform: Envelope}

Formula. Now we derive an important formula which works in rather general context.

Theorem 6.3 (Envelope Formula). Let $\gamma:(a, b) \rightarrow \operatorname{MAT}(\Omega), \gamma(t)=$ $(p(t), r(t))$ be a $C^{1}$ curve such that $\left|p^{\prime}(t)\right|=1$ and $p(t)$ is a 2-prong point for all $t \in(a, b)$. Then the contact set $C(p(t))$ consists of exactly two isolated contact points for $t \in(a, b)$, and their position vectors $x_{+}(t), x_{-}(t)$ are given by

$$
x_{+}(t)=p(t)+r(t)\left\{-r^{\prime}(t) p^{\prime}(t)+\sqrt{1-\left(r^{\prime}(t)\right)^{2}} q(t)\right\}
$$

and

$$
x_{-}(t)=p(t)+r(t)\left\{-r^{\prime}(t) p^{\prime}(t)-\sqrt{1-\left(r^{\prime}(t)\right)^{2}} q(t)\right\},
$$

where $q(t)$ is the vector obtained from rotating $p^{\prime}(t)$ counterclockwise by the right angle.

Proof. Let $x$ be a contact point for $(p(t), r(t))$. Then it must satisfy

$$
|x-p(t)|=r(t) \text { and }|x-p(t+\epsilon)| \geq r(t+\epsilon),
$$


for sufficiently small $|\epsilon|$. Thus if we put

$$
f(\epsilon)=|x-p(t+\epsilon)|^{2}-r(t+\epsilon)^{2},
$$

then the above condition is equivalent to

$$
f(0)=0 \text { and } f(\epsilon) \geq 0,
$$

for sufficiently small $|\epsilon|$. Since $\gamma$ is at least $C^{1}$ near $t, f$ is $C^{1}$ near 0 . Thus the conditions on $f$ imply

$$
f(0)=0 \text { and } f^{\prime}(0)=0
$$

which is equivalent to

$$
|x-p(t)|=r(t)
$$

and

$$
\left\langle x-p(t), p^{\prime}(t)\right\rangle=-r(t) r^{\prime}(t) .
$$

Note that the above equations specifies the length of $x-p(t)$ and the angle between $x-p(t)$ and $p^{\prime}(t)$. From this we can easily see that these two equations have exactly two distinct solutions, and they are $x_{+}(t)$ and $x_{-}(t)$ in Equations(2) and (3) which must be the only contact points for $p(t)$, since $p(t)$ is a 2-prong point.

Remark 6.4. Note the point $x_{+}(t)$ and $x_{-}(t)$ are 'always' in symmetric positions each other with respect to the line through $p(t)$ with the direction of the vector $p^{\prime}(t)$.

Remark 6.5. Let $y_{+}(t)=\left(x_{+}(t)-p(t)\right) / r(t)$ and $y_{-}(t)=$ $\left(x_{-}(t)-p(t)\right) / r(t)$ for $a<t<b$, i.e.,

$$
y_{ \pm}(t)=-r^{\prime}(t) p^{\prime}(t) \pm \sqrt{1-\left(r^{\prime}(t)\right)^{2}} q(t) .
$$

Let $\theta(t)>0$ be the angle between the vectors $y_{ \pm}(t)$ and $p^{\prime}(t)$ and $\alpha(t)$ be the angle between the vectors $\gamma^{\prime}(t)$ and $p^{\prime}(t)$. (Here we let $\alpha(t)$ have the same sign with $r^{\prime}(t)$.)

Then we have

$$
\cos (\theta(t))=-r^{\prime}(t), \quad \sin (\theta(t))=\sqrt{1-\left(r^{\prime}(t)\right)^{2}}
$$

and

$$
\tan (\alpha(t))=r^{\prime}(t) .
$$

Thus we can conclude that

$$
\cos (\theta(t))=-\tan (\alpha(t)) .
$$




\section{Regularity of Medial Axis Transform.}

7.1. Local Regularity of Medial Axis Transform. We now present a sharp local regularity result for the medial axis transform. This kind of regularity result is of fundamental importance in our understanding of the geometric properties of $\operatorname{MAT}(\Omega)$.

Here we will call $\Omega$ a $C^{k}$ domain, if $\Omega$ satisfies all conditions in our standing assumption except that the real analyticity of the boundary is replaced by $C^{k}$.

Theorem 7.1. Let $\Omega$ be a $C^{k}$ domain $(k=1, \ldots, \infty, \omega)$, and let $(p, r)$ be a point in $\mathbf{M A T}(\Omega)$ such that the contact set of $p$ consists of exactly two points $q_{1}$ and $q_{2}$. Suppose the following conditions hold.

(1) If $q_{i}(i=1,2)$ is not a dull corner, then $\partial \Omega$ is $C^{k}$ near $q_{i}$, and $p$ is within the focal locus of a small piece of $\partial \Omega$ near $q_{i}$.

(2) If $q_{i}(i=1,2)$ is a dull corner, then $\overrightarrow{q_{i} p}$ is in a purely interior direction of $q_{i}$.

Then $\mathbf{M A}(\Omega)$ (resp., $\mathbf{M A T}(\Omega))$ is a $C^{k}$ curve near $p($ resp., $(p, r))$.

Proof. By the assumptions, we can take two curves $\gamma_{i}:(-\epsilon, \epsilon) \rightarrow \partial \Omega$, $i=1,2$, near $q_{i}$ respectively, such that, for each $i=1,2, p$ is within the focal locus of $\gamma_{i}$ near $q_{i}$ if $q_{i}$ is not a dull corner. Let $\rho_{i}$ be the distance function from $\gamma_{i}$ for $i=1,2$. By [7], $\rho_{i}$ is a $C^{k}$ function near $p$, if we take sufficiently small $\epsilon>0$. Let $F(x, y)=\rho_{1}(x, y)-\rho_{2}(x, y)$ which is defined near $p$. Then $\nabla F(p)=\nabla \rho_{1}(p)-\nabla \rho_{2}(p)=\frac{\overrightarrow{q_{1} p}}{\left|\overrightarrow{q_{1} p}\right|}-\frac{\overrightarrow{q_{2} p}}{\left|\overrightarrow{q_{2} p}\right|} \neq 0$. Then by the implicit function theorem, there is a line $L$ through $p$ such that, near $p$, the set $M=\left\{(x, y) \in \mathbb{R}^{2} \mid F(x, y)=0\right\}$ is a graph of a $C^{k}$ function over $L$. Note that $L$ can be chosen to be the line passing through $p$ in the direction of $\vec{v}$ which is perpendicular to $\overrightarrow{p q_{1}}-\overrightarrow{p q_{2}}$. From Remark 5.1 , we can see easily that there are no points in $\mathbf{M A}(\Omega)$ other than those in $M$ near $p$. Thus $\mathbf{M A}(\Omega)$ is represented by a $C^{k}$ curve $\alpha$ near $p$. If we let $r(s)$ be such that $(\alpha(s), r(s))$ be in $\operatorname{MAT}(\Omega)$, then $r(s)=\rho_{1}(\alpha(s))$, and hence $r$ is also $C^{k}$. Thus $\operatorname{MAT}(\Omega)$ is a $C^{k}$ curve near $(p, r)$.

Remark 7.1. In general, if $\mathbf{M A}(\Omega)$ goes through the center $p$ of an inscribed osculating circle with contact arc, then $\mathbf{M A}(\Omega)$ may not even be $C^{1}$, even if the boundary is $C^{k}(k \geq 1)$. In Figure 6 below, $\Omega$ is a $C^{1}$ domain, but $\mathbf{M A}(\Omega)$ is only $C^{0}$ near $p$. By changing the boundary of $\Omega$ slightly to make it $C^{\infty}$, we can easily construct an example of a $C^{\infty}$ domain $\Omega$ with $\mathbf{M A}(\Omega)$ not even $C^{1}$. 


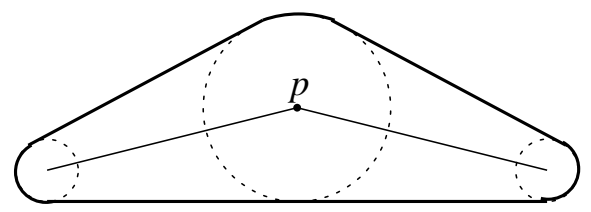

Figure 6.

From Theorem 7.1, we have the following local result.

Corollary 7.1. $\quad$ Let $(p, r)$ be a point in $\mathbf{M A T}(\Omega)$ such that $p \in \mathbf{M A}(\Omega)$ is a generic 2-prong. Then $\mathbf{M A}(\Omega)$ (resp., $\mathbf{M A T}(\Omega)$ ) is a real analytic curve near $p($ resp., $(p, r))$.

7.2. Continuity of Medial Axis Transform Map. Let $\gamma_{1}:(a, b) \rightarrow$ $\partial \Omega^{*}$ be a one-to-one, continuous curve such that $\gamma_{1}(t)$ is a generic point for $t \in(a, b)$. Then by the definition of generic point, $\beta\left(\gamma_{1}(t)\right)$ is a generic 2 prong which we denote by $p(t) \in \mathbf{M A}(\Omega)$ for $t \in(a, b)$. Let $r:(a, b) \rightarrow \mathbb{R}$ be defined such that $(p(t), r(t)) \in \mathbf{M A T}(\Omega)$, and let $\gamma(t)=(p(t), r(t))$ for $t \in(a, b)$. Now, by the definition of generic 2-prong, there exists a unique generic point $\gamma_{2}(t) \in \partial \Omega^{*}$ which is a contact point of $p(t)$ other than $\gamma_{1}(t)$ for $t \in(a, b)$.

Lemma 7.1 (Limit of Generic 2-prongs). Let $\gamma_{1}:(a, b) \rightarrow \partial \Omega^{*}$ be $a$ one-to-one, continuous curve such that $\gamma_{1}(t)$ is a generic point for $t \in(a, b)$, and let $\gamma_{2}, \gamma, p$ and $r$ be defined as above. Then $\gamma_{2}$ and $\gamma$ are also one-toone, continuous curves, and $\gamma_{2}$ has no intersections with $\gamma_{1}$. Furthermore, we have the following.

(1) The lengths of $p$ and $\gamma$ are finite.

(2) $\lim _{t \rightarrow a} p(t)$ and $\lim _{t \rightarrow a} r(t)$ exist, and are denoted by $p(a)$ and $r(a)$ respectively.

(3) $\lim _{t \rightarrow a} p^{\prime}(t)$ and $\lim _{t \rightarrow a} r^{\prime}(t)$ exist, and are denoted by $p^{\prime}(a)$ and $r^{\prime}(a)$ respectively.

(4) $(p(a), r(a))$ is in $\operatorname{MAT}(\Omega)$ and $\gamma_{i}(a), i=1,2$ are contact points of $p(a)$, where $\gamma_{i}(a)=\lim _{t \rightarrow a} \gamma_{i}(t)$ for $i=1,2$.

Proof. It is an easy consequence of Lemma 6.1, Theorem 6.2 and Corollary 7.1 that $\gamma_{2}$ is a one-to-one, continuous curve with no intersections with $\gamma_{1}$. By Corollary 7.1, we may assume $\gamma(t)$ and $p(t)$ are real analytic after some reparametrization of $t$. Furthermore, we take the unit speed parametrization 
for $p$, i.e., $\left|p^{\prime}\right| \equiv 1$. In doing so, we may have to allow $a=-\infty$ or $b=\infty$. Now from Equations (2) and (3) in Theorem 6.3, we have

$$
\frac{1}{2}\left(\frac{x_{+}(t)-p(t)}{r(t)}+\frac{x_{-}(t)-p(t)}{r(t)}\right)=-r^{\prime}(t) p^{\prime}(t)
$$

and

$$
\frac{1}{2}\left(\frac{x_{+}(t)-p(t)}{r(t)}-\frac{x_{-}(t)-p(t)}{r(t)}\right)=\sqrt{1-\left(r^{\prime}(t)\right)^{2}} q(t)
$$

Note that if $x_{ \pm}$are curves in $\partial \Omega$ with no corner points, then the terms $\left(x_{ \pm}(t)-p(t)\right) / r(t)$ are nothing but the unit outer normals of $\partial \Omega$ at $x_{ \pm}(t)$ respectively. If $x_{ \pm}$are dull corners, then $-\left(x_{ \pm}(t)-p(t)\right) / r(t)$ are the interior directions of $x_{ \pm}$respectively. In any case, the limits of the left sides of Equations (4) and (5) exist. Thus $\lim _{t \rightarrow a}\left|r^{\prime}(t) p^{\prime}(t)\right|=\lim _{t \rightarrow a}\left|r^{\prime}(t)\right|$ exists. If $\lim _{t \rightarrow a}\left|r^{\prime}(t)\right|=0$, then by $(5), \lim _{t \rightarrow a} p^{\prime}(t)$ exists, as $p^{\prime}(t)$ is $q(t)$ rotated by 90 degrees clockwise. Suppose $\lim _{t \rightarrow a}\left|r^{\prime}(t)\right|=c>0$ but $\lim _{t \rightarrow a} r^{\prime}(t)$ does not exists. Then by the intermediate value theorem, there is a sequence $t_{n} \rightarrow a$ such that $r^{\prime}\left(t_{n}\right)=0$. Thus by (5), the limit of the norm of the left side of (5) must be 1 , which must be less than 1 since $\lim _{t \rightarrow a}\left(r^{\prime}(t)\right)^{2}>0$. Thus we have a contradiction and $\lim _{t \rightarrow a} r^{\prime}(t)(\neq 0)$ exists. Then by $(4), \lim _{t \rightarrow a} p^{\prime}(t)$ exists.

Now suppose $a=-\infty$. Then since $\left|p^{\prime}\right|=1$ and $\lim _{t \rightarrow a} p^{\prime}(t)$ exists, the curve $p$ cannot be bounded, which is a contradiction to the fact that $p$ is in $\mathbf{M A}(\Omega)$. Similarly $b \neq \infty$. Thus the length of $p$ is finite. Since $\left|r^{\prime}\right| \leq 1$, it follows that the length of $\gamma$ is also finite, and so the existence of the limits $\lim _{t \rightarrow a} p(t)$ and $\lim _{t \rightarrow a} r(t)$ is obvious. That $(p(a), r(a))$ is in $\mathbf{M A T}(\Omega)$ and $\gamma_{i}(a)$ 's correspond to contact points of $p(a)$ is an easy consequence of the fact that $\left(x_{ \pm}(t)-p(t)\right) / r(t)$ converge.

From Lemma 7.1, we can obtain the following theorem.

Theorem 7.2 (Continuity of Medial Axis (Transform) Map). The medial axis transform map $\tilde{\beta}=(p, r): \partial \Omega^{*} \rightarrow \mathbf{M A T}(\Omega)$ and the medial axis map $\beta: \partial \Omega^{*} \rightarrow \mathbf{M A}(\Omega)$ defined in Section 6.1 is continuous.

Proof. Let $t_{0}$ be a point in $\partial \Omega^{*}$. By Theorem 6.2, we can take a small interval $\left(t_{0}-\epsilon, t_{0}+\epsilon\right)$ around $t_{0}$ such that each of the two open intervals $\left(t_{0}-\epsilon, t_{0}\right)$ and $\left(t_{0}, t_{0}+\epsilon\right)$ consists of either only generic points or only points in $\partial \Omega^{*}$ corresponding to points in a circular arc in $\partial \Omega$. If one of the intervals $\left(t_{0}-\epsilon, t_{0}\right)$ and $\left(t_{0}, t_{0}+\epsilon\right)$ corresponds to the latter case, the continuity from 
that interval is obvious. So it is sufficient to show that $\lim _{t \rightarrow t_{0}+} \tilde{\beta}(t)=\tilde{\beta}\left(t_{0}\right)$ and $\lim _{t \rightarrow t_{0}+} \beta(t)=\beta\left(t_{0}\right)$, where we have supposed $\left(t_{0}, t_{0}+\epsilon\right)$ consists of only generic points. But this is an immediate consequence of Lemma 7.1, and thus we have the proof.

7.3. Path-connectedness of Medial Axis Transform. The following theorem is a consequence of the continuity of the medial axis (transform) map in the previous section. In fact, the proof contains a useful argument which is the key idea of the process called killing homology in [2]. See also $[6,11,15]$ for other proofs.

Theorem 7.3. $\mathrm{MAT}(\Omega)$ and $\mathbf{M A}(\Omega)$ is path connected.

Proof. Suppose $\Omega$ has genus $g$. Note that $\operatorname{MAT}(\Omega)=\cup_{i=1}^{g+1} \tilde{\beta}\left(T_{i}\right)$, where $\partial \Omega^{*}=\cup_{i=1}^{g+1} T_{i}$ is defined as in Section 6.1. We now join all the boundary curves $C_{1}, C_{2}, \ldots, C_{g+1}$ by contact circles as follows: Let $C_{1}$ be the outer boundary curve. First, choose an inner boundary curve, say $C_{2}$, which is closest to the outer boundary curve $C_{1}$. Let $q_{1} \in C_{1}$ and $s_{1} \in C_{2}$ be points such that $d\left(q_{1}, s_{1}\right)=d\left(C_{1}, C_{2}\right)$. Draw a disk $B_{r_{1}}\left(p_{1}\right)$ with the segment $\overline{q_{1} s_{1}}$ as the diameter. Then it is easy to see that $B_{r_{1}}\left(p_{1}\right)$ is a maximal disk contained in $\Omega$, i.e., $B_{r_{1}}\left(p_{1}\right) \in \mathbf{C O R E}(\Omega)$. We recursively define $B_{r_{i}}\left(p_{i}\right)$ as follows: Suppose $\left\{C_{1}, C_{2}, \ldots, C_{i}\right\}$ are joined by disks $B_{r_{1}}\left(p_{1}\right), \ldots, B_{r_{i-1}}\left(p_{i-1}\right)$ in $\operatorname{CORE}(\Omega)$. Choose a curve, say $C_{i+1}$, among unselected ones $\left\{C_{i+1}, \ldots\right.$, $\left.C_{g+1}\right\}$ such that $C_{i+1}$ is closest to the set $C_{1} \cup \cdots \cup C_{i}$. Let $q_{i} \in C_{1} \cup \cdots \cup C_{i}$ and $s_{i} \in C_{i+1}$ be points such that $d\left(q_{i}, s_{i}\right)=d\left(C_{i+1}, \cup_{k=1}^{i} C_{k}\right)$. Then the disk $B_{r_{i}}\left(p_{i}\right)$ with the segment $\overline{q_{i} s_{i}}$ as the diameter is again in $\operatorname{CORE}(\Omega)$.

Note the continuity of $\tilde{\beta}$ implies that each $\tilde{\beta}\left(T_{i}\right)$ is path-connected, and since $\left(p_{i}, r_{i}\right) \in\left(\tilde{\beta}\left(T_{i}\right) \cap \tilde{\beta}\left(T_{i+1}\right)\right) \neq \emptyset$ for $1 \leq i \leq g, \operatorname{MAT}(\Omega)=\cup_{i=1}^{g+1} \tilde{\beta}\left(T_{i}\right)$ itself is path connected. The path connectedness of $\mathbf{M A}(\Omega)$ follows easily from that of $\mathbf{M A T}(\Omega)$.

Remark 7.2. $\quad$ It is easy to see that the disk $B_{r_{i}}\left(p_{i}\right)$ connecting one of $C_{1}, \ldots, C_{i}$ to $C_{i+1}$ does not make contact with any other boundary curve. Therefore, this process of connecting the boundary curves stops in exactly $g$ step, where $g$ is the genus of $\Omega$.

\section{Graph Structure of Medial Axis Transform.}

8.1. Topology of Medial Axis Transform. One of the nice features of the medial axis (transform) is that it preserves the topological information of the domain. We illustrate this fact by showing that $\mathbf{M A}(\Omega)$ is a strong 
deformation retract of $\Omega$, hence $\mathbf{M A}(\Omega)$ and $\Omega$ are homotopically equivalent. For a definition of strong deformation retract, see [12].

Let $p$ be any point in the interior $\operatorname{int}(\Omega)$ of $\Omega$, and $q$ be a point in $\partial \Omega$ such that $d(p, \partial \Omega)=d(p, q)$. Suppose $p \notin \mathbf{M A}(\Omega)$. Then the point $q$ is unique. Let $m(q)$ be the point in $\mathbf{M A}(\Omega)$ such that $q$ is a contact point of $m(q)$ and $p$ is on the line segment $\overline{q m(q)}$. We now define the homotopy $H_{\delta}: \operatorname{int}(\Omega) \rightarrow \mathbf{M A}(\Omega) \backslash\{$ sharp corners $\}$ for $\delta \in[0,1]:$ If $p \notin \mathbf{M A}(\Omega)$, then define $H_{\delta}(p)=p(\delta)$, where $p(\delta)$ is a point in the line segment between $p$ and $m(q)$ such that the ratio of the length of $\overline{p(\delta) m(q)}$ over the length of $\overline{p m(q)}$ is $1-\delta$. If $p \in \mathbf{M A}(\Omega)$, simply define $H_{\delta}(p)=p$. We call $H_{\delta}$ the canonical homotopy associated with the medial axis. That $H_{\delta}$ is a continuous map is an easy consequence of the fact that the medial axis map $\beta$ is a continuous map from $\partial \Omega^{*}$ to $\mathbf{M A}(\Omega)$ which is Theorem 7.2. Therefore we have proved the following

Theorem 8.1. $\mathbf{M A}(\Omega) \backslash\{$ sharp corners $\}$ is a strong deformation retract of int $(\Omega)$ by the canonical homotopy defined above.

Now let $\Omega_{\delta}=\overline{H_{\delta}(\operatorname{int}(\Omega))}$. Then for sufficiently small $\delta$, it is trivial to define a homotopy $R_{\delta}$ retracting $\Omega$ to $\Omega_{\delta}$ while keeping $\mathbf{M A}(\Omega)$ fixed. Composing $R_{\delta}$ with $H_{\delta}$ above, we have

Corollary 8.1. $\mathbf{M A}(\Omega)$ is a strong deformation retract of $\Omega$. Hence $\mathbf{M A}(\Omega)$ (and thus $\mathbf{M A T}(\Omega)$ ) is homotopic to $\Omega$.

Remark 8.1. In [15], F.-E. Wolter obtained the same result using different method. It should be noted his result is also valid for higher dimension when the boundary is smooth. Sherbrooke, Patrikalakis and Wolter also obtained the same result [11].

8.2. Geometric Graph. In this section we summarize the results obtained so far to show that the medial axis and the medial axis transform have the structure which we will call geometric graph. The graph structure of medial axis (transform) is extremely useful. It allows us to store and process the entire information of a domain which is a 2-dimensional object, as a form of graph which is 1-dimensional and relatively easy to handle.

We will call a point $p \in \mathbf{M A}(\Omega)$ a vertex, if $p$ is not a generic 2-prong. So the number of the vertices of $\mathbf{M A}(\Omega)$ is finite by Theorem 6.2. Let $p \in \mathbf{M A}(\Omega)$ be a generic 2-prong. Then by Theorem 6.2 and Corollary 7.1, it is easy to see that there exists a maximal real analytic curve in $\mathbf{M A}(\Omega)$ containing $p$ such that all points on it are generic 2-prongs. We call such a curve in $\mathbf{M A}(\Omega)$ an edge. Thus every generic 2-prong is in one of the edges 
of $\mathbf{M A}(\Omega)$ and the set of generic points in $\mathbf{M A}(\Omega)$ is the union of all the edges. Especially the edges have no mutual intersections and each edge has no self-intersections. Now Lemma 7.1 says that each edge has a finite length and its end points must be vertices. So an edge is one of the following type:

(1) A curve connecting two vertices.

(2) A curve whose both end points coincide at one vertex.

(3) A closed curve.

Let $p \in \mathbf{M A}(\Omega)$ be a vertex which is an $n$-prong point $(n \geq 1)$. Then there are $n$ points (or intervals) $C_{1}, \ldots, C_{n}$ in $\partial \Omega^{*}$ which correspond to the $n$ contact components of $p$ respectively, and there are $2 n$ open intervals of generic points in $\partial \Omega^{*}$ each of whose one end point is in $\cup_{i=1}^{n} C_{i}$. By Corollary 7.1 and Lemma 7.1, these intervals are paired to produce exactly $n$ real analytic curves emanating from $p$. This means that the vertex degree of $p$ is $n$. Since the number of the vertices is finite and the number of the contact components of each vertices are finite by Lemma 4.1, it follows that the number of edges is also finite. Finally, note that $\mathbf{M A}(\Omega)$ is connected by Theorem 7.3.

The above arguments can be applied in the same way to $\operatorname{MAT}(\Omega)$, and we skip the analogous definitions and results here. We call a set in $\mathbb{R}^{2}$ (or in $\mathbb{R}^{3}$ ) a geometric graph, if it is topologically a usual connected graph with finite number of vertices and edges, where a vertex is a point in $\mathbb{R}^{2}$ (or in $\mathbb{R}^{3}$ ) and an edge is a real analytic curve with finite length whose limits of tangents at the end points exist.

Summarizing the above arguments, we have:

\section{Theorem 8.2 (Graph Structure of Medial Axis (Transform)).}

$\operatorname{MA}(\Omega)(\mathbf{M A T}(\Omega))$ is a geometric graph, Moreover, we have the following.

(1) A point in an edge of $\mathbf{M A}(\Omega)$ is a generic 2-prong, and hence $\mathbf{M A}(\Omega)$ is real analytic at this point.

(2) A vertex of degree 1 in $\mathbf{M A}(\Omega)$ is either a sharp corner or the center of an inscribed osculating circle with one contact component.

(3) A vertex of degree 3 or higher in $\mathbf{M A}(\Omega)$ is a bifurcation point.

(4) A vertex of degree 2 in $\mathbf{M A}(\Omega)$ is a 2-prong which is not generic.

Remark 8.2. Note that $\mathbf{M A}(\Omega)$ and $\mathbf{M A T}(\Omega)$ are isomorphic as graphs. So we have omitted the obvious statements about $\mathbf{M A T}(\Omega)$ in Theorem 8.2.

By using Remark 6.4 and Lemma 7.1, 2-prongs which are not generic can be classified according to whether $\mathbf{M A}(\Omega)$ and $\mathbf{M A T}(\Omega)$ are $C^{1}$ there. 
Let $(p, r)$ be in $\mathbf{M A T}(\Omega)$ such that $p$ is a 2-prong which is not generic.

Type 1. Both of the two contact components of $p$ are isolated contact points:

In this case, $\mathbf{M A}(\Omega)$ and $\mathbf{M A T}(\Omega)$ are both $C^{1}$ at $p$ and $(p, r)$ respectively. This is valid even when the maximal disk $B_{r}(p)$ is osculating $\partial \Omega$ at the contact points, which is illustrated in Figure 7.

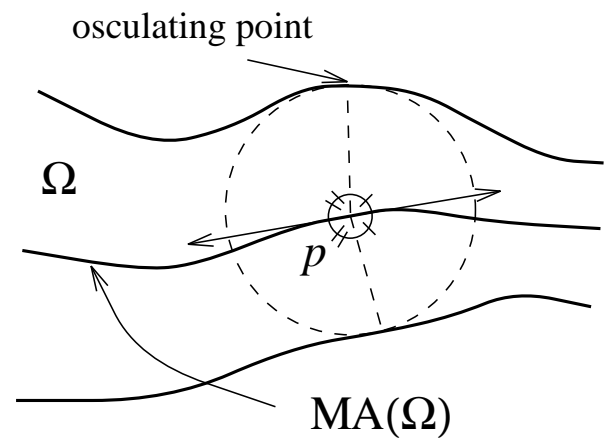

Figure 7. $\mathbf{M A}(\Omega)$ and $\operatorname{MAT}(\Omega)$ are $C^{1}$.

Type 2. At least one contact component of $p$ is a contact arc and the lengths of the two contact components are not same:

Here we consider the length of an isolated contact point to be 0 of course. In this case, both $\mathbf{M A}(\Omega)$ and $\mathbf{M A T}(\Omega)$ are not $C^{1}$ at $p$ and $(p, r)$ respectively. See Figure 8.

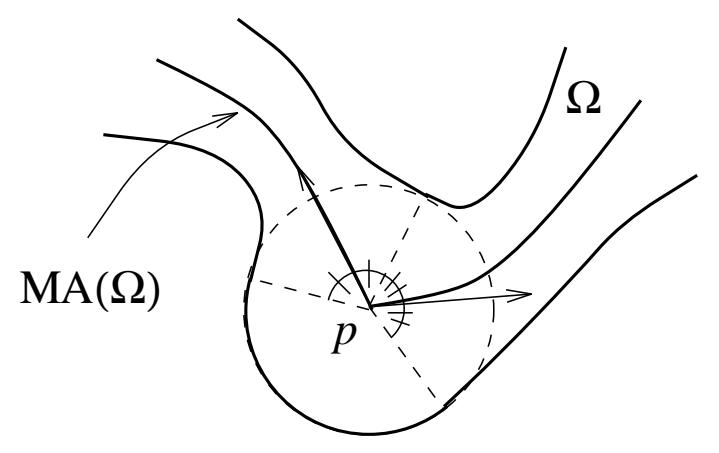

Figure 8. $\mathbf{M A}(\Omega)$ and $\operatorname{MAT}(\Omega)$ are not $C^{1}$.

Type 3. Both of the two contact components are contact arcs with the same length:

In this case, while $\mathbf{M A T}(\Omega)$ is still not $C^{1}$ at $(p, r), \mathbf{M A}(\Omega)$ is $C^{1}$ at $p$. See Figure 9. 


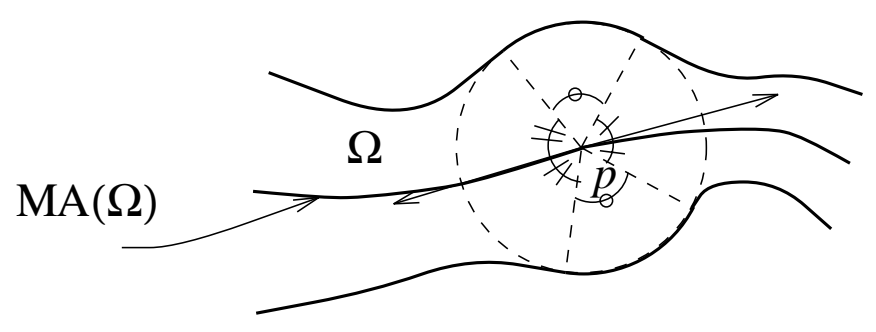

Figure 9. $\mathbf{M A}(\Omega)$ is $C^{1}$ but $\operatorname{MAT}(\Omega)$ has a $C^{1}$ break.

\section{Concluding Remarks.}

Throughout this paper, the real analyticity condition on the boundary of a domain is used to prove various finiteness results: The number of the contact components of a medial axis point, the number of the 1-prongs, the number of the bifurcation points, and the number of the medial axis points which are not generic 2-prongs. We remark that a domain satisfying these finiteness conditions has the same properties concerning the medial axis (transform) with the ones in our class, even if the boundary is only $C^{k}$ $(k=2, \ldots, \infty)$ rather than real analytic. But as one can see, usually these finiteness conditions cannot easily be checked in general.

\section{References}

[1] H. Blum, A transformation for extracting new descriptors of shape. In W.W. Dunn, editor, Proc. Symp. Models for the Perception of Speech and Visual Form, pp. 362380. MIT Press, Cambridge, MA, USA, 1967.

[2] H.I. Choi, S.W. Choi, H.P. Moon and N.-S. Wee, New algorithm for medial axis transform of plane domain, preprint.

[3] G.L. Dirichlet, Über die Reduktion der positiven quadratischen Formen mit drei unbestimmten ganzen Zahlen, J. of Reine Angew. Math., 40 (1850), 209-227.

[4] S.M. Gelston and D. Dutta, Boundary surface recovery from skeleton curves and surfaces, Computer Aided Geometric Design, 12 (1995), 27-51.

[5] M. Held, On the computational geometry of pocket machining, Vol. 500 of Lecure Notes in Computer Science, Springer-Verlag, 1991.

[6] C. M. Hoffmann and C.-S. Chiang, The medial axis transform for $2 D$ regions, preprint.

[7] S.G. Krantz and R.P. Harold, Distance to $C^{k}$ hypersurfaces, J. of Differential Equations, 40 (1981), 116-120.

[8] D.T. Lee, Medial axis transform of a planar shape, IEEE Trans. Pattern Analysis and Machine Intelligence, PAM, I-4(4) (1982), 363-369. 
[9] D.T. Lee and R.L. Drysdale, Generalization of Voronoi diagrams in the plane, SIAM J. of Computing, 10 (1981), 73-87.

[10] F.P. Preparata and M.I. Shamos, Computational geometry an introduction, Springer-Verlag, 1985.

[11] E.C. Sherbrooke, N.M. Patrikalakis and F.-E. Wolter, Differential and topological properties of medial axis transforms, to appear in Graphical Models and Image Processing.

[12] E.H. Spanier, Algebraic topology, McGraw-Hill, 1966.

[13] V. Srinivasan and L.R. Nackman, Voronoi diagram for multiply connected polygonal domains I: Algorithm, IBM J. of Res. Develop., 31(3) (1987), 361-372.

[14] G.M. Voronoi, Nouvelles applications des paramètres continus à la théorie des formes quadratiques. Recherches sur les parallélloèdres primitifs, J. Reine Angew. Math., 134 (1908), 198-287.

[15] F.-E. Wolter, Cut locus and medial axis in global shape interrogation and representation, Technical report.

[16] C.K. Yap, An $O(n * \log n)$ algorithm for the Voronoi diagram of a set of simple curve segments, Discrete and Computational Geometry, 2(4) (1987), 365-393.

Received March 18, 1996. All authors are supported in part by BSRI and GARC, and the second author is also supported in part by Daewoo Foundation.

Seoul National University

SEOUl, 151-742 KoreA

E-mail address: hichoi@math.snu.ac.kr

Seoul National University

SEOUl, 151-742 Korea

E-mail address: swchoi@math.snu.ac.kr

AND

Seoul National University

SEOUl, 151-742 KoreA

E-mail address: hpmoon@math.snu.ac.kr 